\title{
Title
}

\section{Localised expression of OsIAA29 suggests a key role for auxin in regulating development of the dorsal aleurone of early rice grains.}

\section{Authors}

Mafroz A. Basunia ${ }^{\mathrm{a}, \mathrm{b}}$, Heather M. Nonhebel ${ }^{\mathrm{a}}$, David Backhouse ${ }^{\mathrm{c}}$ and Mary McMillan ${ }^{\mathrm{a}}$.

\section{Affiliations}

${ }^{a}$ School of Science and Technology, University of New England, Armidale, New South Wales 2351, Australia

${ }^{\mathrm{b}}$ Present address, Department of Biochemistry and Molecular Biology, Jahangirnagar University, Dhaka 1342, Bangladesh

${ }^{c}$ School of Environmental and Rural Science, University of New England, Armidale, New South Wales 2351, Australia

\section{Correspondence}

H. M. Nonhebel; Email, hnonheb2@une.edu.au; Phone, +61 267732083

\section{ORCID IDs}

MAB 0000-0002-3433-2615, HMN 0000-0001-9055-3095, DB 0000-0003-0663-6002, MM 00000002-2336-3985

\section{Main Conclusion}

Non-canonical AUX/IAA protein, OsIAA29, and ZmMPR-1 homologues, OsMRPLs, are part of an auxin-related signalling cascade operating in the dorsal aleurone during early rice grain development.

\section{Abstract}

Endosperm of rice and other cereals accumulates high concentrations of the predominant in planta auxin, indole-3-acetic acid (IAA) during early grain development. However, IAA signalling and function during endosperm development are poorly understood. Here, we report that OSYUC12 (an auxin biosynthesis gene) and OsIAA29 (encoding a non-canonical AUX/IAA) are both expressed exclusively in grains, reaching a maximum 5 to 6 days after pollination. OsYUC12 expression is localized in the aleurone, sub-aleurone and embryo, whereas OsIAA29 expression is restricted to a narrow strip in the dorsal aleurone, directly under the vascular bundle. Although rice has been reported to lack endosperm transfer cells (ETCs), this region of the aleurone is enriched with sugar transporters and is likely to play a key role in apoplastic nutrient transfer, analogous to ETCs in other cereals. OSIAA29 has orthologues only in grass species; expression of which is also specific to early grain development. OSYUC12 and OsIAA29 are temporally co-expressed with two genes (AL1 and OsPR602) previously linked to the development of dorsal aleurone or ETCs. Also up regulated at the 
same time are a cluster of MYB-related genes (designated OsMRPLs) homologous to ZmMRP-1, which regulates maize ETC development. Wheat homologues of ZmMRP-1 are also expressed in ETCs. Although previous work has suggested that other cereals do not have orthologues of ZmMRP1, our work suggests OsIAA29 and OsMRPLs and their homologues in other grasses are part of an auxin-regulated, conserved signalling network involved in the differentiation of cells with ETC-like function in developing cereal grains.

\section{Key words}

Dorsal aleurone, auxin, endosperm, ZmMRP-1, OsYUC12, OsIAA29 


\section{Introduction}

The yield and quality of cereal grains is dependent on the coordinated regulation of endosperm development, uptake of photosynthate and production of storage molecules, and is highly susceptible to adverse environmental influences ( $\mathrm{Yu}$ et al. 2015). In addition, the molecular and physiological events taking place during early endosperm development determine to a large extent the final grain size and weight in rice and other cereals (Mizutani et al. 2010; Fahy et al. 2018). There is a large body of literature reporting the involvement of plant hormones in the regulation of early endosperm development as well as its response to environment (reviewed by Basunia and Nonhebel 2019). However, little is known of the detailed role of hormonal signalling and how this influences key processes of endosperm cellularisation, differentiation of cells responsible for nutrient uptake or the expression of starch and storage protein synthesis genes.

Previous work in our laboratory has shown that a large increase in the auxin, indole-3-acetic acid (IAA), occurs during endosperm cellularisation, aleurone development and the initiation of starch production in rice grains, driven by strong up-regulation of key auxin biosynthesis genes, OsTAR1, OsYUC9, OsYUC11 and OsYUC12 (Abu-Zaitoon et al. 2012; Russell French et al. 2014; Nonhebel and Griffin 2020). This work identified small differences in the expression profiles of $O_{s} Y U C 9, O_{s} Y U C 11$ and $O_{s} Y U C 12$, suggesting that some subfunctionalisation may occur. OsYUC12 was found to be exclusively expressed in endosperm for a short period between approximately four and seven days after pollination (DAP). The up- and down-regulation of OSYUC12 also appeared to coincide with that of OSIAA29, encoding an atypical AUX/IAA protein that may play a role as a transcriptional co-regulator in auxin signalling.

A co-expression analysis using OsYUC12 and OsIAA29 as bait genes, and online accessed microarray data from several experiments, revealed a small group of genes with the same expression profile restricted to the endosperm from approximately 3 to 7 DAP (Nonhebel and Griffin 2020). This included genes that are exclusively expressed in the dorsal aleurone of rice, i.e. OsPR602, OsPR9a, $A L 1$ and $O S N F-Y B 1$ (at the early stage of expression) (Li et al. 2008; Kuwano et al. 2011; Xu et al. 2016). Of these, $O S N F-Y B 1$ is reported to play a key role in endosperm development; plants in which the gene was down-regulated had small grains with chalky endosperm (Xu et al. 2016). The other dorsal aleurone-specific genes, OsPR602, OsPR $9 a$ and $A L 1$ have been reported to have promoters that direct expression to endosperm transfer cells ( $\mathrm{Li}$ et al. 2008) or contained cis-elements similar to those interacting with 
ZmMRP-1 (Kuwano et al. 2011), an atypical MYB transcription factor that regulates development of endosperm transfer cells (ETCs) in maize (Zea mays) (Gómez et al. 2009). The co-expressed gene group also contained a previously un-reported cluster of MYB-related transcription factor-like genes from rice that are the closest rice homologues to ZmMRP-1. These data suggested a possible auxin-signalling network associated with development of cells responsible for uptake of nutrients into the developing endosperm.

Investigations of the role of IAA in rice grain development have been limited by a lack of mutants. More extensive work has been carried out on its importance for the development of maize endosperm, where mutants, defective endosperm18 (de18) and defective kernel18 (dek18) have reduced expression of IAA biosynthesis genes, reduced IAA content as well as an aberrant basal endosperm transfer layer (BETL) and small, shrivelled grains (Bernardi et al. 2012, 2016). In maize grains, IAA accumulates specifically in the BETL and aleurone (Forestan et al. 2010). Furthermore, treatment of developing maize grains with the auxin transport inhibitor, N-1-naphthylphthalamic acid (NPA), resulted in the formation of a multilayered aleurone instead of a single layer, suggesting that an IAA maximum at the endosperm periphery acts as a signal for aleurone differentiation. The most recent work by Bernardi et al. 2019 investigated the transcriptome of de18 maize grains. Their results suggest that ZmMRP1 as well as genes that are regulated by this transcription factor are down-regulated in the auxin deficient mutant. Thus, IAA may regulate BETL development in maize by controlling expression of ZmMRP-1.

We have previously reported evidence for conservation of auxin signalling networks operating during grain development of different cereals (Russell French et al. 2014). Rice does not have a well-defined ETC layer (Hands et al. 2012). However, the dorsal aleurone is likely to play a similar role in apoplastic nutrient transfer to the developing endosperm given its proximity to the vascular trace and enrichment with sugar transporters (Bai et al. 2016; Xu et al. 2016). Based on our previous observations in rice as well as the information from maize, we suggest that IAA may regulate the development of the dorsal aleurone in rice. We therefore hypothesise that expression of OSYUC12 and OsIAA29 may be localised to these cells. We tested this hypothesis by investigating, via in situ hybridisation, the localisation of OsYUC12 and OsIAA29 and comparing this with the previously studied and dorsal aleuronespecific $O S P R 602$. To identify precisely the timing of maximum expression of OSYUC12 and OsIAA29 as well as test their temporal co-expression with OsPR602, OsPR9a, AL1 and three rice homologues of $Z m M R P-1$, here designated as rice MRP-1-like or OsMRPL. (OsMRPL1, 
OsMRPL3 and OSMRPL4), we carried out a quantitative expression study from grain samples harvested at daily intervals from 1 to 10 DAP.

The existence of a conserved signalling network within cereals, by which auxin regulates the development of ETCs or cells with ETC-like properties, requires the presence in other cereals of orthologues of key proteins with the same expression profile. As Hands et al. (2012) have previously reported the absence of any orthologues of ZmMRP-1 in other cereals, we investigated the phylogeny and protein structures of ZmMRP-1 and its closest homologues in maize, rice, wheat (Triticum aestivum) and Brachypodium distachyon. Expression of the wheat MRP-like genes was investigated for comparison, using the Wheat Expression Browser (Borrill et al. 2016; Ramírez-González et al. 2018) which has a large number of samples including from dissected grains. A similar phylogenetic and in silico expression analysis was carried out on putative cereal orthologues of OsIAA29. Finally, we investigated whether OsIAA29-like proteins are restricted to cereals or whether they occur in dicots and non-grass monocots.

\section{Materials and Methods}

\section{Plant material and growing conditions}

Rice plants (Oryza sativa ssp. japonica cv. Reiziq) were grown in a greenhouse at the University of New England under natural light with $30^{\circ} \mathrm{C} / 18^{\circ} \mathrm{C}$ day/night temperatures. Seven rice seeds were sown directly into flooded cylindrical plastic pots $(50 \mathrm{~cm} \times 15 \mathrm{~cm})$ filled with cracking clay soil (vertosol). When the seedlings reached the 2-3 leaf growth stage, they were thinned to three plants per pot. Plants were watered daily and fertilised fortnightly with the commercial fertiliser Aquasol $^{\circledR}(2.0 \mathrm{gm} / \mathrm{L})$ until panicle initiation. Panicles in which approximately half of the spikelets reached anthesis were tagged in the afternoon. The date of tagging was recorded as the day of pollination; the following day was designated as 1 day after pollination (DAP) and so on. Tagged panicles were harvested daily in the afternoon from 1 to 10 DAP. Only superior caryopses were collected, weighed, frozen immediately in liquid nitrogen and stored at $-80^{\circ} \mathrm{C}$ until further use.

\section{RNA extraction, reverse transcription and quantitative real-time PCR}

Total RNA was extracted from 80-100 mg grain samples using Bioline ${ }^{\circledR}$ ISOLATE II RNA Plant Kit (Meridian Bioscience). RNA concentration and purity were measured by NanoDrop ${ }^{\text {TM }} 8000$ Spectrophotometer (Thermo Fisher Scientific). RNA quality was checked by the presence of two clear bands of $18 \mathrm{~S}$ and $28 \mathrm{~S}$ rRNAs following agarose gel 
electrophoresis (Nolan et al. 2006). Only high-quality RNA with A260/A280 ratio in the range of 1.8-2.0 was used in downstream applications.

Transcript sequences of OsYUC12, OsIAA29, AL1, OsPR602, OsPR9a, OsMRPL1, OSMRPL3 and OSMRPL4 were downloaded from PHYTOZOME 12.0 (Goodstein et al. 2012). Primer pairs were designed using Primer3 software (Koressaar et al. 2018) (Supplementary Table S1). Either the left or the right primer of each gene was designed to span an exon-exon boundary in order to avoid amplification of any residual genomic DNA contaminant. Amplification of a single product of the expected size by a primer pair was first confirmed by RT-PCR using Qiagen ${ }^{\circledR}$ One-Step RT-PCR kit (Qiagen) followed by agarose gel analysis of the amplified products. The gene for rice ubiquitin-conjugating enzyme E2 (OsUBC; LOC_OsO2g42314) was used as the reference gene (Li et al. 2010).

Quantitative real-time RT-PCR was done in two steps. Bioline ${ }^{\circledR}$ SensiFAST $^{\mathrm{TM}}$ cDNA Synthesis Kit (Meridian Bioscience) was used to synthesize cDNA from $1.0 \mu \mathrm{g}$ of total RNA template per reaction according to manufacturer's instructions. A no-RT control that contained all reaction components except the reverse transcriptase was also included. Bioline $^{\circledR}$ SensiFAST $^{\mathrm{TM}}$ SYBR $^{\circledR}$ No-ROX Kit (Meridian Bioscience) was used for the quantitative PCR. Each well of a 96-well plate contained $10 \mathrm{ng}$ of cDNA per $20 \mu \mathrm{l}$ of final reaction volume. All other reagents were added as per the manufacturer's instructions. A notemplate control was included as negative control. Three biological replicates and two technical replicates were included for each primer set. Reactions were carried out in CFX96 Touch $^{\mathrm{TM}}$ Real-Time PCR Detection System (Bio-Rad Laboratories). The amplification program used was as follows: $95^{\circ} \mathrm{C}$ for $2 \mathrm{~min}$ and 40 cycles of $95^{\circ} \mathrm{C}$ for $5 \mathrm{~s}, 60^{\circ} \mathrm{C}$ for $10 \mathrm{~s}$ and $72^{\circ} \mathrm{C}$ for $5 \mathrm{~s}$. Melt curve analysis confirmed the amplification of a single uniform product by each primer pair. This was further confirmed by an agarose gel electrophoresis. Primers with poor amplification efficiency or melt curve analysis implying the presence of more than one product were replaced with new primer sets and the experiment repeated. The manufacturer's software was used to calculate the expression of the targeted genes relative to the expression of the reference gene. Data from the two technical replicates were first averaged, then the mean and standard error of the three biological replicates from the same developmental stage were calculated. 


\section{In situ mRNA hybridisation}

In situ mRNA hybridisation was carried out using the protocol of Drews 1998 with some modifications. Immature rice grains collected at 5, 6 and 7 DAP were trimmed at both ends, and their palea and lemma were carefully removed. Trimmed grains were immediately fixed in freshly prepared formalin-acetic acid-alcohol (FAA; 3.7\% formaldehyde, 5\% acetic acid and $50 \%$ ethanol) fixative first under gentle vacuum on ice for $15 \mathrm{~min}$ and then overnight at $4^{\circ} \mathrm{C}$. The fixed grains were dehydrated by a graded ethanol series and xylene before infiltrating them with paraffin wax (Paraplast Plus) in an automated tissue processor (TP1020, Leica Biosystems). The grains were embedded in paraffin wax on an embedding centre (EG1150, Leica Biosystems). Paraffin sections $(8.0 \mu \mathrm{m}$ thick) were cut using a rotary microtome (RM2235, Leica Biosystems) and transferred to glass slides coated with poly-Llysine (Sigma-Aldrich). The slides were air-dried overnight and stored at $4^{\circ} \mathrm{C}$ until further use.

Purified cDNAs from OsYUC12, OsIAA29 and PR602 templates were cloned into pGEM ${ }^{\circledR}$-T vector (Promega) following manufacturer's instructions (refer to Supplementary Table S2 for primer pairs used to amplify the templates). Gene inserts were amplified from the plasmid by T7 and SP6 primers which annealed to T7 and SP6 promoters flanking the inserts. Purified DNA amplicons with flanking T7 and SP6 promoters were used for in vitro synthesis of digoxigenin (DIG)-UTP-labelled single-stranded RNA sense and anti-sense probes by using T7 and SP6 polymerases from a DIG RNA Labelling Kit (Roche). The non-complementary sense probe was used as negative control for each gene. The probes were hydrolysed with $200 \mathrm{mM}$ carbonate buffer at $60^{\circ} \mathrm{C}$ for $80-90$ min to generate $150-200 \mathrm{bp}$ fragments.

Selected paraffin sections were de-waxed with Histoclear (Sigma-Aldrich), and rehydrated with a graded ethanol series and PBS. They were treated with $1.0 \mu \mathrm{g} / \mathrm{mL}$ proteinase $\mathrm{K}$ solution for $30 \mathrm{~min}$ at $37^{\circ} \mathrm{C}$. The sections were dehydrated with a graded ethanol series and probes were applied (320 ng probe in $100 \mu \mathrm{l}$ hybridisation buffer per slide). Hybridisation was carried out overnight in a humidified box at $55^{\circ} \mathrm{C}$. After a series of washes, immunodetection of the DIG-labelled probes was carried out by using an anti-DIG antibody coupled with alkaline phosphatase and a ready-to-use 5-bromo-4-chloro-3-indolyl phosphate (BCIP)/nitro-blue tetrazolium (NBT) solution (Sigma-Aldrich) as the chromogenic substrate for the enzyme. The slides were mounted with an aqueous medium made of glycerol and TE buffer $(50 \% \mathrm{v} / \mathrm{v})$. Images of the sections were acquired by a high-definition slide scanner (NanoZoomer 2.0-RS, Hamamatsu Photonics). 


\section{Phylogenetic analysis and database mining}

BLASTP search (Altschul et al. 1997) was conducted using OsIAA29 and ZmMRP-1 peptides as queries against proteomes of selected monocots and eudicots on PHYTOZOME 12.0 (Goodstein et al. 2012) and Ensembl Plants 47 (Kersey et al. 2016). Members of the CCA1-like subgroup of MYB-related transcription factors were selected based on information in Du et al. (2013). Molecular Evolutionary Genetics Analysis (MEGA) version $X$ (Kumar et al. 2018) was used for the phylogenetic analysis. The sequences were aligned by MUSCLE (Edgar, 2004). Phylogenetic trees were constructed by the Maximum Likelihood method based on JTT matrix-based model (Jones et al. 1992). The reliability of each node in the tree was determined by the bootstrap test from 500 replicates (Felsenstein 1985). OsIAA29 orthologues were compared with homologues from bamboo and non-grass monocots by multiple sequence alignment using Clustal Omega (https://www.ebi.ac.uk). RNA-seq database of Rice Genome Annotation Project (Kawahara et al. 2013) was used to analyse expression profiles of rice genes. Expression data of OSIAA29 and OsMRPL orthologues in wheat and barley (Hordeum vulgare) were retrieved from online RNA-seq databases, Wheat Expression Browser http://www.wheat-expression.com (Borrill et al. 2016; Ramírez-González et al. 2018) and BaRTv1.0 https://bio.tools/BaRTv1.0 (Rapazote-Flores et al. 2019), respectively. Co-expression and cis-regulatory element (CRE) analyses were conducted as described previously by Nonhebel and Griffin (2020).

\section{Results}

\section{Gene expression during the first 10 days after pollination}

The expression of OsIAA29 and OsYUC12 was compared with that of OsPR602, OsPR $9 a$, $A L 1$ and three OsMRPLs (OsMRPL1, OsMRPL3 and OsMRPL4) in immature grains from 1 to 10 DAP by qRT-PCR. All genes showed a somewhat similar expression profile (Fig. 1). In particular, all genes except OSMRPL3 were not expressed until 2 to 3 DAP and expression was maximal at 5 to 6 DAP, before declining to low levels by 9 DAP. OSMRPL3 followed a similar pattern of up-regulation followed by down-regulation, but this was earlier, with expression detected at $1 \mathrm{DAP}$ and maximal expression at 4 to 5 DAP. OSMRPL4 had the most restricted expression, with very little transcript detected apart from 5- and 6-DAP samples. On the other hand, $A L 1$ still continued to be active at 10 DAP. As gene expression was only examined in developing grain samples, global expression of genes in other parts of the plant was investigated using RNA-seq datasets available via the Rice Genome Annotation Project. As shown in Fig. 2, expression was mostly restricted to early grain development with 
no gene activity found in vegetative tissues, floral tissues, or more mature grains at 25 DAP. However, OsMRPL3, OsMRPL4 and OSMRPL5 did show slight expression in anthers.

\section{Localisation of OsPR602, OsIAA29 and OsYUC12 expression in early grains}

Spatial expression of OsPR602, OsIAA29 and OsYUC12 was examined in immature rice grains by in situ mRNA hybridisation. The results reported are from sections of grains collected at 7 DAP, just past the peak of expression, as the larger grains were easier to section and gene expression was still detectable. The sense probes of the genes tested did not give any hybridisation signal, validating the experimentation. Signal from the anti-sense probe of OsPR602 confirmed its spatial expression in the dorsal aleurone (Fig. $3 \mathrm{~b}-\mathrm{c}$ and $\mathrm{f}-\mathrm{g}$ ). The spatial expression of OSIAA29 was similar to that of OsPR602, with its hybridisation signal restricted exclusively to the dorsal aleurone (Fig. $4 \mathrm{~b}-\mathrm{c}$ and $\mathrm{f}-\mathrm{g}$ ). The expression of OsIAA29 was confined to a narrow strip directly under the major vascular bundle (Fig. $4 \mathrm{f}-\mathrm{g}$ ). We did not detect any signal for OsIAA29 expression in the ventral aleurone, starchy endosperm, embryo (Fig. 4d), pericarp and vascular bundles. Spatial expression of OsYUC12, on the other hand, followed a much broader pattern than that of OSPR602 and OSIAA29. As shown by both longitudinal and transverse sections, its hybridisation signal was detected in the aleurone and sub-aleurone layers; the signal was distributed both in the dorsal and ventral sides of aleurone (Fig. 5 b-c and f-g). We also detected the hybridisation signal in the embryo (Fig. 5d). However, there was no signal in the starchy endosperm, pericarp and vascular bundles.

\section{Phylogenetic analyses of $O S I A A 29$ and $O S M R P L s$ and expression of orthologues in other cereals}

As little is known about the non-canonical AUX/IAA protein OsIAA29 or any of its orthologues, we carried out a comprehensive search for, and phylogenetic analysis of similar proteins to explore whether this protein was restricted to cereals. A BLASTP search on PHYTOZOME 12.0 (Goodstein et al. 2012) using OsIAA29 as query against proteomes of selected species found proteins with high homology in both cereal and non-cereal grass species, excepting sequences from moso bamboo (Phyllostachys edulis J.Houz.) which showed low peptide homology. Amino acid identity was also low for sequences from nongrass monocots and eudicots. The phylogenetic tree comparing all AUX/IAA proteins from rice with closest homologues from other monocots and eudicots (Fig. 6) confirmed that OsIAA29 had putative orthologues in all grass species tested except moso bamboo. No putative orthologues were found in eudicots. The multiple sequence alignment shown in 
Suppl. Fig. S1 showed that, like OsIAA29, putative orthologues lacked the N-terminal domains I and II of canonical AUX/IAA proteins and had an acidic C-terminal extension. On the other hand, closest homologous peptides from moso bamboo, banana and the sea grass Zostera marina showed typical AUX/IAA domain structure. We explored the expression of barley and wheat orthologues of OSIAA29 via publicly available RNA-seq databases. The barley orthologue is expressed exclusively in immature grains at 5 DAP, with no expression recorded in embryo at 4 DAP or other tissues (Suppl. Fig. S2A). The expression of wheat orthologues is also restricted to early grain/endosperm around 10 DAP (Suppl. Fig. S2B).

Apart from our previous report of OsMPRL genes, no orthologues of ZmMRP-1 have been described. Fig. 7 shows a phylogenetic analysis of all members of the Circadian Clock Associated 1-like (CCA1-like) sub-group of MYB-related transcription factors from maize, rice and $B$. distachyon as well as wheat proteins homologous to ZmMRP-1. The phylogram showed a well-supported clade (bootstrap value of 85) containing ZmMRP-1 and five closely related maize proteins, as well as OsMRPL1-5, four proteins from B. distachyon and 21 wheat homologues. As MYB-related transcription factors are highly diverse, the reliability of these relationships was tested by using other methods of phylogenetic analysis: Neighbour Joining (Saitou and Nei 1987) and Minimum Evolution (Rzhetsky and Nei 1992). All analyses reliably grouped the OsMRPLs with ZmMRP-1. All species investigated had multiple paralogues, existing as tandem repeats indicating a high level of recent, lineagespecific gene expansion. OsMRPL1 and OsMRPL2 as well as OsMRPL4 and OsMRPL5 were pairs of tandem duplicates. Although the tree suggests that OsMRPLs as well as proteins from wheat and $B$. distachyon are potentially orthologous to ZmMRP-1, the amino acid identity between OsMRPLs and ZmMRP-1 is very low, ranging from 30 to 38\%. However, the multiple sequence alignment (ClustalO) shown in Fig 8 demonstrates homology between ZmMRP-1 and putative orthologues in rice, wheat and B. distachyon in the N-terminal domain as well as the MYB domain. It is also noteworthy that ZmMRP-1 itself is missing part of this N-terminal domain compared with other homologues in maize as shown by the inclusion of the sequence encoded by GRMZM2G121111_T01. Although all OSMRPL genes appeared to be expressed, a detailed investigation of the peptide sequences (Suppl. Fig. S3) indicated that OsMRPL5 lacked a section of the N-terminal domain found in OsMRPL4 and other proteins. It also had a large insert within the MYB domain. In addition, a comparison of tandem duplicates OsMRPL1 and OsMRPL2 indicated a number of short indels between them. 
To investigate possible orthologous expression of MRPL proteins within the grains of another cereal, we used the Wheat Expression Browser as we were unsuccessful in obtaining in situ hybridisation results for the OSMRPLs. The results shown in Fig. 9 demonstrated expression of 11 out of the 21 wheat $M R P L$ genes. These were active exclusively in developing grains with significant expression seen in whole grain samples only at 10 DAP, coinciding with expression of wheat co-orthologues of OSIAA29. Although expression in whole grains was very low at 20 DAP, in manually dissected samples, high relative expression was seen exclusively in the endosperm transfer cell (ETC) layer, indicating highly localised upregulation of these genes. Supplementary Fig. S4 compares expression of ZmMRP-1 and other full-length members of this clade in maize with that of ZmIAA38, the putative orthologue of OSIAA29. As with the other cereals there is a strong co-expression seen with maximal expression at 12 DAP.

\section{Promoter analysis of genes co-expressed with OsIAA29, OsPR602 and OsMRPLs}

The existence of putative orthologues of ZmMRP-1 in rice and other cereals with similar expression profiles suggested conservation of function of these proteins. ZmMRP-1 interacts with a 12-bp cis-acting regulatory element (CRE) consisting of two tandem repeats of TATCTC (TC-box) in the promoter region of its target genes (Barrero et al. 2006). With a view to examining the putative enrichment of this CRE in promoter regions of rice genes, we first determined the genes co-expressed with OsIAA29, OsMRPL1 and OsPR602, and ranked them as described previously by Nonhebel and Griffin 2020. We then searched for statistically significant enrichment of the CRE in 1000-bp promoter regions of top 300 coexpressed genes, using the RiceFREND platform (Sato et al. 2013). The 12-bp CRE was not found. However, a single TATCTC element was significantly enriched on both promoter strands of the top 100 co-expressed genes (Table 1). Enrichment was strongly associated with co-expression rank.

\section{Discussion}

In this study, we aimed to investigate IAA biosynthesis and signalling during crucial early stages of endosperm development in rice by exploring in detail the temporal and spatial expression of putative IAA biosynthesis and signalling genes, viz. OsYUC12 and OsIAA29, respectively. Spatial expression was compared with that of OsPR602, a gene that has previously been reported as expressed exclusively in the dorsal aleurone of developing rice grains. The precise timing of up-regulation was also compared with that of a group of genes previously suggested (Nonhebel and Griffin 2020) to be co-expressed with OSIAA29 and 
OsYUC12, i.e. OsPR9a, $A L 1$ and three homologues of ZmMRP-1 that we have designated as OsMRPLs.

Our results confirmed that all genes were co-expressed, except for OsMRPL3 which was upregulated a little earlier. Expression was grain-specific and short-lived, with genes most active between 3 and 7 DAP. Their up-regulation thus coincides with initiation of coenocyte cellularisation, its completion and differentiation of the early aleurone/starchy endosperm, which take place at 3 DAP, 5 DAP and 7 DAP, respectively (Wu et al. 2016). This supports their possible involvement in an auxin-related signalling pathway during a key stage of transcriptional reprogramming associated with coenocyte cellularisation and differentiation of the early aleurone and starchy endosperm.

Several genes confirmed as temporally co-expressed with OSIAA29 have localised expression within the dorsal aleurone in rice (Li et al. 2008; Kuwano et al. 2011). Here, we have also demonstrated dorsal aleurone-specific expression of OsIAA29. This part of rice aleurone lying adjacent to the major vascular bundles is enriched with sugar transporters and may play an important role in apoplastic nutrient transfer into developing grains (Bai et al. 2016; Xu et al. 2016). Unlike maize, wheat and barley, rice aleurone lacks a well-defined ETC layer (Hands et al. 2012). However, a number of observations point to an ETC-like identity and activity of the dorsal aleurone. Dorsal aleurone-specific OsPR602 has orthologues in barley (END1) and wheat (TaPR60), which are also expressed only in ETCs (Doan et al. 1996; Li et al. 2008; Kovalchuk et al. 2009). A GUS-reporter gene coupled with OsPR602 promoter was expressed exclusively in transgenic barley ETCs (Li et al. 2008). Spatio-temporal expression pattern of OsIAA29 is almost identical to that of OsPR602. This suggests a role for the atypical AUX/IAA protein encoded by this gene in signalling during differentiation of dorsal aleurone cells that regulate apoplastic nutrient transfer from maternal pericarp into developing endosperm.

The temporal co-expression of rice homologues of $Z m M R P-1$ provides further evidence for a signalling node regulating development of cells with an ETC-like function. Hands et al (2012) have previously reported that no orthologues of ZmMRP-1 could be identified in temperate cereals or in rice. They further observed that expression of the closest homologue in B. distachyon (Bradilg72300) could not be detected in developing grain tissue by reverse transcriptase PCR. The very high sequence diversity seen between MYB-related transcription factors makes it very difficult to predict the existence of orthologues of ZmMRP-1. However, our phylogenetic and multiple sequence analyses of homologues of CCA1-like sub-group of 
MYB-related transcription factors does indicate the existence of a well-supported clade of similar proteins in rice, wheat and $B$. distachyon. Our expression analysis via a combination of reverse transcriptase PCR and RNA-seq data also shows that activity of rice and wheat homologues is restricted to a short period during early grain development and furthermore that expression in wheat is highest or possibly restricted to ETCs. The short-lived nature of expression may have led to lack of detection of Bradi1g72300 by Hands et al. (2012). It is also of note that all MRPLs appear to have undergone several episodes of gene expansion, with evidence of recent tandem duplication as well as earlier duplication events. Not all genes in each species are expressed; this could also explain the lack of detectable expression of Bradilg72300. ZmMRP-1 transactivates BETL-specific genes by interacting with a TC-box motif consisting of two 6-bp tandem repeats (TATCTCTATCTC) in their promoter (Barrero et al. 2006). Publications on AL1 (Kuwano et al. 2011), OsPR602 and OsPR9a (Li et al. 2008) have already suggested that regulation of gene expression within the dorsal aleurone of rice may be regulated in a conserved manner in cereals. Supporting this we found significant enrichment of the TC-box like motif, TATCTC (one of the tandem repeats found in maize) within the promoter regions of genes co-expressed with OSIAA29, OsPR602 and OSMRPL1. Future research focusing on the question of whether OsMRPLs bind to this cis-element and regulate gene expression should shed more light on their functions during early endosperm development.

In maize, a study of gene expression in the auxin-deficient de18 mutant by Bernardi et al. (2019) has provided evidence for the regulation of ZmMRP-1 and other members of the gene cluster by IAA. The temporal co-expression of OSMRPLs with OsYUC12 and OsIAA29 suggests that this regulation may be conserved across different cereals. We have previously suggested that three rice YUCCA genes predominantly expressed in grains but with differing patterns of temporal expression point to sub-functionalisation and highly localised IAA production at different stages of endosperm development (Basunia and Nonhebel 2019). We hypothesised that the IAA fraction contributed by OsYUC12 may regulate the molecular events occurring during early stages of endosperm development such as coenocyte cellularisation and early aleurone differentiation. Further support for this view came from its spatial expression pattern. In situ hybridisation signal from OsYUC12 transcripts was detected in the aleurone and sub-aleurone at 7 DAP (Fig. 5), suggesting localised biosynthesis of IAA in early aleurone. In separate work from our laboratory (Kabir et al. 2020), we have also recently reported highest expression of wheat auxin biosynthesis genes 
TaYUC9-B1 and TaYUC9D1 in aleurone and transfer cells although other genes TaYUC10-A and TaYUC10-D were most highly expression in the starchy endosperm. Forestan et al. 2010 reported strong IAA signal in the aleurone and BETL of maize kernels; ZmYUC1 expression occurs predominantly in maize aleurone (Zhan et al. 2015; Doll et al. 2017). All results indicate the production of IAA within the aleurone layer of developing cereals grains. Furthermore, OsYUC12 expression appears to be regulated by two MADS-box transcription factors, MADS78 and MADS79 (Paul et al. 2020). Their expression peaks at 2 DAP and becomes suppressed by 4 DAP. According to the model of Paul et al., they inhibit OsYUC12 transcription, allowing thereby the proliferation of the coenocyte nuclei. OsYUC12 transcription is triggered when MADS78 and MADS79 expression is suppressed, allowing biosynthesis of IAA which mediates the transition and progression from the coenocytic to the cellularised endosperm. A critical level of endogenous IAA is also required for the cellularisation to proceed in Arabidopsis endosperm (Batista et al. 2019).

Although the in situ results for OsYUC12 and OsIAA29 suggest the production and function of IAA in the dorsal aleurone, the role of OsIAA29 within an auxin signalling pathway is unclear as this protein and its orthologues in other cereals lacks the $\mathrm{N}$-terminal region required for interaction with the TIR1-like auxin receptor. IAA33, a non-canonical AUX/IAA from Arabidopsis lacking the degron and EAR domains like $O s$ IAA29, negatively regulates auxin signalling in root tip to maintain root distal stem cell identity (Lv et al. 2020). IAA33 competes with the canonical IAA5 for binding to ARF10 and ARF16. OsIAA29 may function in a similar way by competing with a canonical $O s$ AUX/IAA protein for binding to one or more, as yet unidentified, OsARFs. OsIAA29 has orthologues only in cereal and noncereal grass species. Surprisingly, given the close phylogenetic relationship between the subfamilies Oryzoideae and Bambusoideae, no orthologue was detected in moso bamboo. However, over the course of evolution, bamboo species have acquired enormous morphological variation, including different caryopses (Ruiz-Sanchez and Sosa 2015). The ancestral gene for OsIAA29 was probably lost in moso bamboo. Published reports on the expression and function of OsIAA29 orthologues are scarce. The sorghum orthologue is expressed in immature grains at 4 DAP (Nonhebel and Griffin 2020). Analysis of RNA-seq databases indicate early grain- or endosperm-specific expression of the barley, wheat and maize orthologues (Figs. S2 and S4). This gene thus appears to have evolved independently in the grass family (Poaceae) and acquired a novel and grain-specific function during early endosperm development. 
In conclusion, we have confirmed the temporal co-expression of OsYUC12 and OsIAA29 with genes that have an association with ETC-like cells, AL1, OsPR602, OsPR9a, OsMRPL1 and OSMRPL4. In addition, we have shown that expression of OSIAA29 is restricted to a narrow strip in the dorsal aleurone very similar to that reported for OsPR602 and OsPR 9 . We suggest this gene may act with OsMRPLs to regulate cell differentiation in this region. Further work is required to confirm the localisation of OsMRPLs as well as details of the signalling pathway. Although $O_{S} Y U C 12$ expression appeared to occur over a broader region of the aleurone to OSIAA29, our data suggest the production of auxin within the aleurone as has been observed in maize. Future studies focusing on protein-protein interactions of OsIAA29 with OsAUX/IAAs and OsARFs, and targeted inactivation of these genes will provide more information on auxin signalling during early endosperm development.

\section{References}

Abu-Zaitoon YM, Bennett K, Normanly J, Nonhebel HM (2012) A large increase in IAA during development of rice grains correlates with the expression of tryptophan aminotransferase OsTARI and a grain-specific YUCCA. Physiol Plant 146:487-499.

Altschul SF, Madden TL, Schäffer AA, Zhang J, Zhang Z, Miller W, Lipman DJ (1997) Gapped BLAST and PSI-BLAST: a new generation of protein database search programs. Nucleic Acids Res 25:3389-3402.

Bai AN, Lu XD, Li DQ, Liu JX, Liu CM (2016) NF-YB1-regulated expression of sucrose transporters in aleurone facilitates sugar loading to rice endosperm. Cell Res 26:384388.

Barrero C, Muñiz LM, Gómez E, Hueros G, Royo J (2006) Molecular dissection of the interaction between the transcriptional activator ZmMRP-1 and the promoter of BETL-1. Plant Mol Biol 62:655-668.

Basunia MA, Nonhebel HM (2019) Hormonal regulation of cereal endosperm development with a focus on rice (Oryza sativa). Funct Plant Biol 46:493-506.

Batista RA, Figueiredo DD, Santos-González J, Köhler C (2019) Auxin regulates endosperm cellularisation in Arabidopsis. Genes Dev 33:466-476.

Bernardi J, Lanubile A, Li QB, Kumar D, Kladnik A, Cook SD, Ross JJ, Marocco A, Chourey PS (2012) Impaired auxin biosynthesis in the defective endosperm 18 mutant is due to mutational loss of expression in the $\mathrm{ZmYucl}$ gene encoding endospermspecific YUCCA1 protein in maize. Plant Physiol 160:1318-1328.

Bernardi J, Li QB, Gao Y, Zhao Y, Battaglia R, Marocco A, Chourey PS (2016) The auxindeficient defective kernel18 (dek18) mutation alters the expression of seed-specific biosynthetic genes in maize. J Plant Growth Regul 35:770-777. 
Bernardi J, Battaglia R, Bagnaresi P, Lucini L, Marocco A (2019) Transcriptomic and metabolomic analysis of $\mathrm{ZmYUCl}$ mutant reveals the role of auxin during early endosperm formation in maize. Plant Sci 281:133-145.

Borrill P, Ramirez-Gonzalez R, Uauy C (2016). expVIP: a customizable RNA-seq data analysis and visualization platform. Plant Physiol 170:2172-2186.

Doan DN, Linnestad C, Olsen OA (1996) Isolation of molecular markers from the barley endosperm coenocyte and the surrounding nucellus cell layers. Plant Mol Biol 31:877-886.

Doll NM, Depège-Fargeix N, Rogowsky PM, Widiez T (2017) Signaling in early maize kernel development. Mol Plant 10:375-388.

Drews GN (1998) In situ hybridisation. In: Martinez-Zapater JM, Salinas J (eds) Arabidopsis Protocols, Methods in Molecular Biology ${ }^{\mathrm{TM}}$, Volume 82, Humana Press, New York, pp. 353-371.

Du H, Wang YB, Xie Y, Liang Z, Jiang SJ, Zhang SS, Huang YB, Tang YX (2013) Genomewide identification and evolutionary and expression analyses of MYB-related genes in land plants. DNA Res 20:437-448.

Edgar RC (2004) Muscle: Multiple sequence alignment with high accuracy and high throughput. Nucleic Acids Res 32:1792-1797.

Fahy B, Siddiqui H, David LC, Powers SJ, Borrill P, Uauy C, Smith, AM. (2018). Final grain weight is not limited by the activity of key starch-synthesising enzymes during grain filling in wheat. J Exp Bot 69: 5461-5475.

Felsenstein J (1985) Confidence limits on phylogenies: An approach using the bootstrap. Evolution 39:783-791.

Forestan C, Meda S, Varotto S (2010) ZmPIN1-mediated auxin transport is related to cellular differentiation during maize embryogenesis and endosperm development. Plant Physiol 152:1373-1390.

Gómez E, Royo J, Muñiz LM, Sellam O, Paul W, Gerentes D, Barrero C, López M, Perez P, Hueros G (2009) The maize transcription factor Myb-Related Protein-1 is a key regulator of the differentiation of transfer cells. Plant Cell 21:2022-2035.

Goodstein DM, Shu S, Howson R, Neupane R, Hayes RD, Fazo J, Mitros T, Dirks W, Hellsten U, Putnam N, Rokhsar DS (2012) Phytozome: a comparative platform for green plant genomics. Nucleic Acids Res 40:D1178-D1186.

Hands P, Kourmpetli S, Sharples D, Harris RG, Drea S (2012) Analysis of grain characters in temperate grasses reveals distinctive patterns of endosperm organization associated with grain shape. J Exp Bot 63:6253-6266.

Jones DT, Taylor WR, Thornton JM (1992) The rapid generation of mutation data matrices from protein sequences. Bioinformatics 8:275-282.

Kawahara Y, de la Bastide M, Hamilton JP, Kanamori H, McCombie WR, Ouyang S, Schwartz DC, Tanaka T, Wu J, Zhou S, Childs KL, Davidson RM, Lin H, QuesadaOcampo L, Vaillancourt B, Sakai H, Lee SS, Kim J, Numa H, Itoh T, Buell CR, Matsumoto T (2013) Improvement of the Oryza sativa Nipponbare reference genome using next generation sequence and optical map data. Rice 6:4. 
Kabir MR, Backhouse D, Winter G, Nonhebel HM (2020) Expression of TaTAR2.3-1B, TaYUC9-1 and TaYUC10 correlates with auxin and starch content of developing wheat grains. bioRxiv. https://doi.org/10.1101/2020.10.12.336560

Kersey PJ, Allen JE, Armean I, Boddu S, Bolt BJ, Carvalho-Silva D, Christensen M, Davis P, Falin LJ, Grabmueller C, Humphrey J (2016) Ensembl Genomes 2016: more genomes, more complexity. Nucleic Acids Res 44:D574-D580.

Koressaar T, Lepamets M, Kaplinski L, Raime K, Andreson R, Remm M (2018) Primer3_masker: integrating masking of template sequence with primer design software. Bioinformatics 34:1937-1938.

Kovalchuk N, Smith J, Pallotta M, Singh R, Ismagul A, Eliby S, Bazanova N, Milligan AS, Hrmova M, Langridge P, Lopato S (2009) Characterization of the wheat endosperm transfer cell-specific protein TaPR60. Plant Mol Biol 71:81-98.

Kumar S, Stecher G, Li M, Knyaz C, Tamura K (2018) MEGA X: molecular evolutionary genetics analysis across computing platforms. Mol Biol Evol 35:1547-1549.

Kuwano M, Masumura T, Yoshida KT (2011) A novel endosperm transfer cell-containing region-specific gene and its promoter in rice. Plant Mol Biol 76:47 -56.

Li M, Singh R, Bazanova N, Milligan AS, Shirley N, Langridge P, Lopato S (2008) Spatial and temporal expression of endosperm transfer cell-specific promoters in transgenic rice and barley. Plant Biotech J 6:465-476.

Li QF, Sun SSM, Yuan DY, Yu HX, Gu MH, Liu QQ (2010) Validation of candidate reference genes for the accurate normalization of real-time quantitative RT-PCR data in rice during seed development. Plant Mol Biol Rep 28:49-57.

Lv B, Yu Q, Liu J, Wen X, Yan Z, Hu K, Li H, Kong X, Li C, Tian H, De Smet I, Zhang XS, Ding Z (2020) Non-canonical AUX/IAA protein IAA33 competes with canonical AUX/IAA repressor IAA5 to negatively regulate auxin signaling. EMBO J 39:e101515.

Mizutani M, Naganuma T, Tsutsumi K, Saitoh Y (2010) The syncytium specific expression of the Orysa;KRP3 CDK inhibitor: implication of its involvement in the cell cycle control in the rice (Oryza sativa L.) syncytial endosperm. J Exp Bot 61:791-798.

Nolan T, Hands RE, Bustin SA (2006) Quantification of mRNA using real-time RT-PCR. Nat Protoc 1:1559-1582.

Nonhebel HM, Griffin K (2020) Production and roles of IAA and ABA during development of superior and inferior rice grains. Funct Plant Biol 47:716-726.

Paul P, Dhatt BK, Miller M, Folsom JJ, Wang Z, Krassovskaya I, Liu K, Sandhu J, Yu H, Zhang C, Obata T, Staswick P, Walia H (2020) MADS78 and MADS79 are essential regulators of early seed development in rice. Plant Physiol 182:933-948.

Ramírez-González R, Borrill P, Lang D, Harrington SA, Brinton J, Venturini L et al. (2018). The transcriptional landscape of hexaploid wheat across tissues and cultivars. Science 361:eaar6089.

Rapazote-Flores P, Bayer M, Milne L, Mayer CD, Fuller J, Guo W, Hedley PE, Morris J, Halpin C, Kam J, McKim SM, Zwirek M, Casao MC, Barakate A, Schreiber M, Stephen G, Zhang R, Brown JWS, Waugh R, Simpson CG (2019) BaRTv1.0: an 
improved barley reference transcript dataset to determine accurate changes in the barley transcriptome using RNA-seq. BMC Genomics 20:968.

Ruiz-Sanchez E, Sosa V (2015) Origin and evolution of fleshy fruit in woody bamboos. Mol Phylogenet Evol 91:123-134.

Russell French S, Abu-Zaitoon Y, Uddin MM, Bennett K, Nonhebel HM (2014) Auxin and cell wall invertase related signalling during rice grain development. Plants 3:95 -112.

Rzhetsky A and Nei M (1992) A simple method for estimating and testing minimum evolution trees. Mol Biol Evol 9:945-967.

Saitou N, Nei M (1987) The neighbor-joining method: a new method for reconstructing phylogenetic trees. Mol Biol Evol 4:406-425.

Sato Y, Namiki N, Takehisa H, Kamatsuki K, Minami H, Ikawa H, Ohyanagi H, Sugimoto K, Itoh JI, Antonio BA, Nagamura Y (2013) RiceFREND: a platform for retrieving co-expressed gene networks in rice. Nucleic Acids Res 41:D1214-D1221.

Stelpflug, SC, Sekhon RS, Vaillancourt B, Hirsch CN, Buell CR, de Leon N, Kaeppler SM (2016) An expanded maize gene expression atlas based on RNA sequencing and its use to explore root development. Plant Genome 9:1.

Wu X, Liu J, Li D, Liu CM (2016) Rice caryopsis development II: Dynamic changes in the endosperm. J Integr Plant Biol 58:786-798.

Xu JJ, Zhang XF, Xue HW (2016) Rice aleurone layer specific OsNF-YB1 regulates grain filling and endosperm development by interacting with an ERF transcription factor. $J$ Exp Bot 67:6399-6411.

Yu SM, Lo SF, Ho TH (2015) Source-sink communication: regulated by hormone, nutrient, and stress cross-signaling. Trends Plant Sci 20:844-857.

Zhan J, Thakare D, Ma C, Lloyd A, Nixon NM, Arakaki AM, Burnett WJ, Logan KO, Wang D, Wang X, Drews GN, Yadegari R (2015) RNA sequencing of laser-capture microdissected compartments of the maize kernel identifies regulatory modules associated with endosperm cell differentiation. Plant Cell 27:513-531. 


\section{Tables}

Table 1. Enrichment of TC-box like TATCTC element in 1000-bp promoter regions of genes coexpressed with OSIAA29, OSPR602 and OSMRPL1. Statistical significance was calculated using Chisquared tests by comparison to the frequency of this element in all genes (Sato et al. 2013). Its significant enrichment in promoter strands of top 100 co-expressed genes is shown in bold.

\begin{tabular}{lll}
\hline \multicolumn{1}{c}{ Gene sample } & $\begin{array}{c}\text { TATCTC } \\
\text { sense strand }\end{array}$ & $\begin{array}{c}\text { TATCTC } \\
\text { antisense strand }\end{array}$ \\
\hline Top 100 co-expressed genes (RiceFREND) & $\mathbf{3 8 \%} \mathbf{( P = \mathbf { 0 . 0 0 9 } )}$ & $\mathbf{3 8 \%} \mathbf{( P = \mathbf { 0 . 0 0 6 } )}$ \\
Co-expressed genes ranked 101-200 (RiceFREND) & $35 \%(P=0.03)$ & $27 \%(P=0.44)$ \\
Co-expressed genes ranked 201-300 (RiceFREND) & $22 \%(P=0.78)$ & $28 \%(P=0.32)$ \\
All genes (RiceFREND) & $24 \%$ & $23 \%$ \\
\hline
\end{tabular}




\section{Figures}
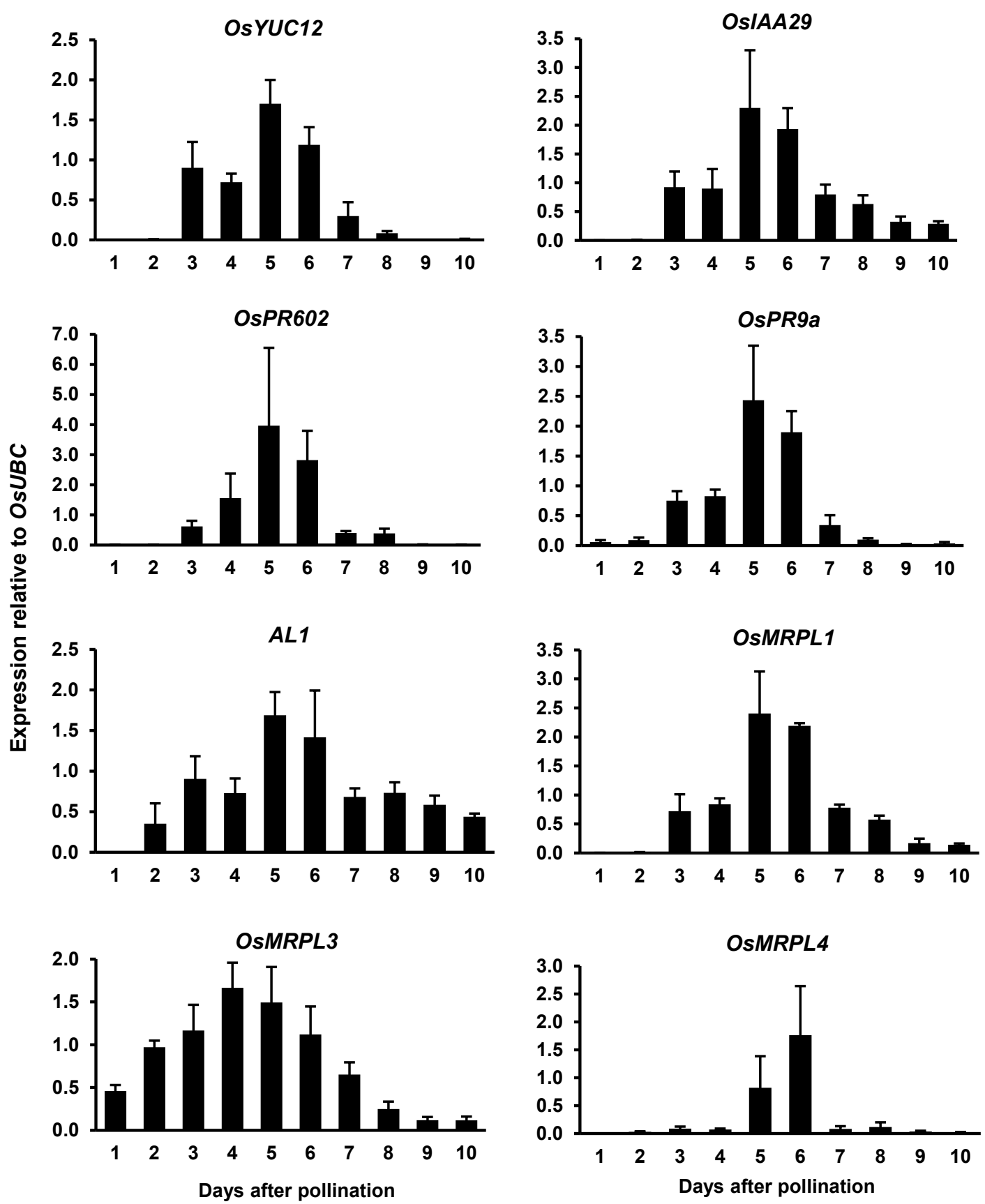

Fig. 1. Expression of OsYUC12, OsIAA29, OsPR602, OsPR9a, AL1, OsMRPL1, OsMRPL3 and OSMRPL4 in whole rice grains from 1 to 10 days after pollination (DAP). Expression of the genes was calculated relative to the expression level of the reference gene OsUBC (LOC_Os02g42314), using the software provided by the manufacturer of the CFX96 Touch $^{\mathrm{TM}}$ Real-Time PCR Detection System (Bio-Rad Laboratories). Results shown are the means \pm the standard errors of the mean (SEMs) of three biological replicates. 


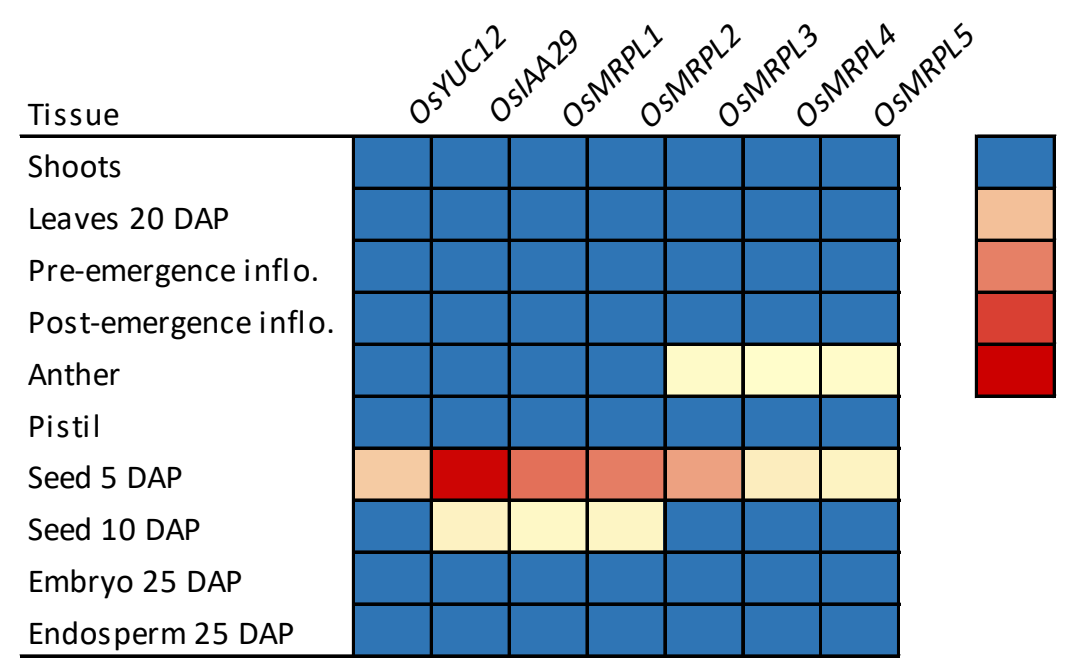

Fig 2. Heat map showing expression of OsYUC12, OsIAA29 and OsMRPL1-5 in different vegetative and reproductive tissues of rice. The expression profiles were retrieved from RNA-seq datasets available on Rice Genome Annotation Project (Kawahara et al. 2013). FPKM = Fragments Per Kilobase of transcript per Million mapped reads. 

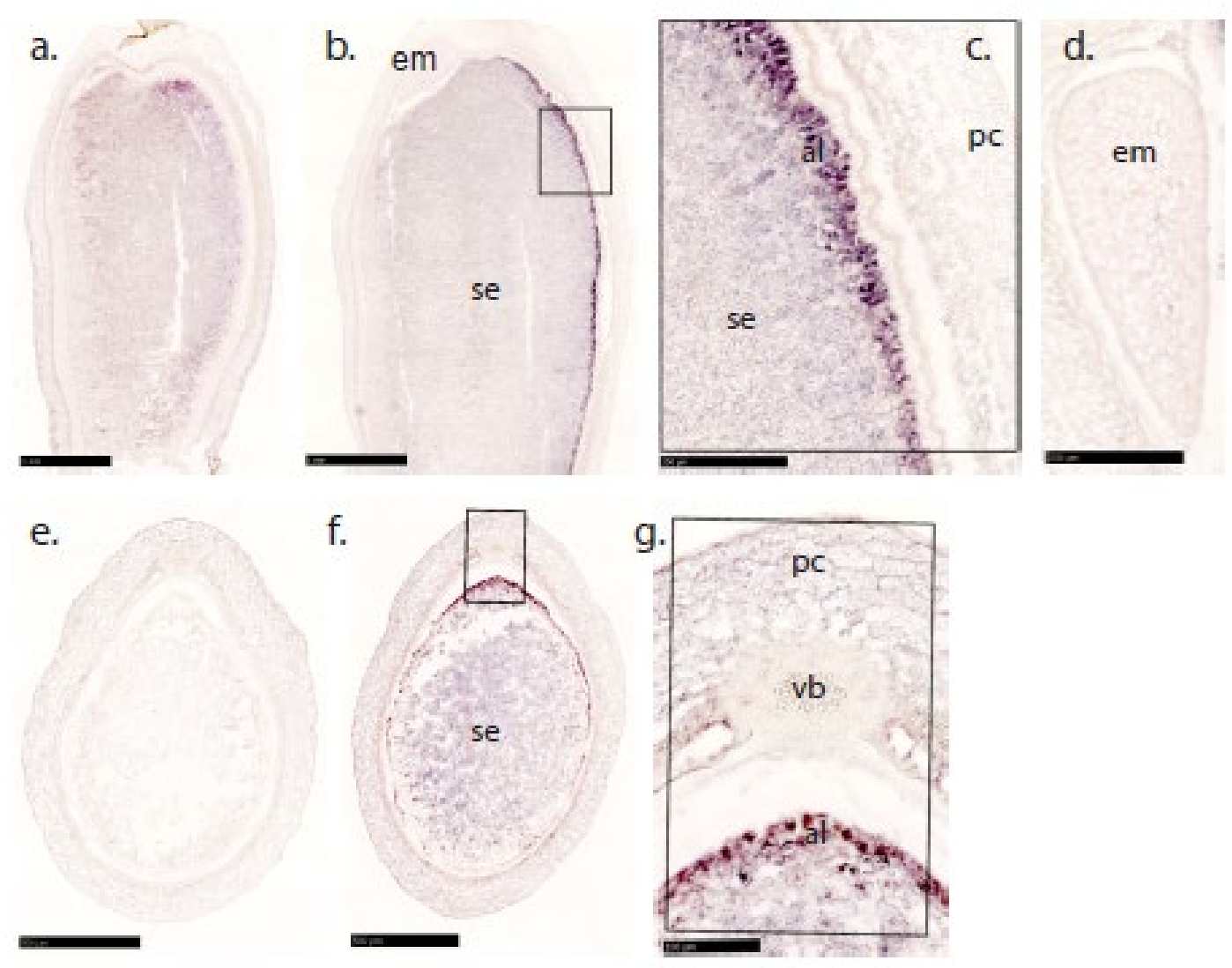

Fig. 3 (a-g). In situ hybridisation of OsPR602 transcripts in immature rice grains. Hybridisation was done using longitudinal (a-d) and transverse sections (e-g) of immature rice grains at 7 DAP. Images show the absence of in situ hybridisation signal from the sense, (a and e) probes and its presence from the anti-sense ( $b-d$ and $f-g$ ) probes. Image $c$ and $g$ are magnified images of marked areas from $b$ and $f$, respectively. Image $d$ is derived from the same section as $b$ and shows the absence of hybridisation signal in the embryo. Sense probes (a and e) were used as negative control in the experimentation. Note the strong hybridisation signal in the dorsal aleurone (b-c and $f-g$ ). The dorsal side in longitudinal grain sections can be determined by the position of the major vascular bundles running in the pericarp along the dorsal side or by the position of the embryo located always on the ventral side. In case of transverse sections, the dorsal side is determined by the position of the major vascular bundles. Scale bars: (a and b) $1000 \mu \mathrm{m}$; (c and d) $250 \mu \mathrm{m}$; (e and f) $500 \mu \mathrm{m}$; (g) $100 \mu \mathrm{m}$. Abbreviations used for annotation: $\mathrm{al}=$ aleurone; $\mathrm{em}=$ embryo; $\mathrm{pc}=$ pericarp; $\mathrm{se}=$ starchy endosperm; $\mathrm{vb}=$ vascular bundle. 

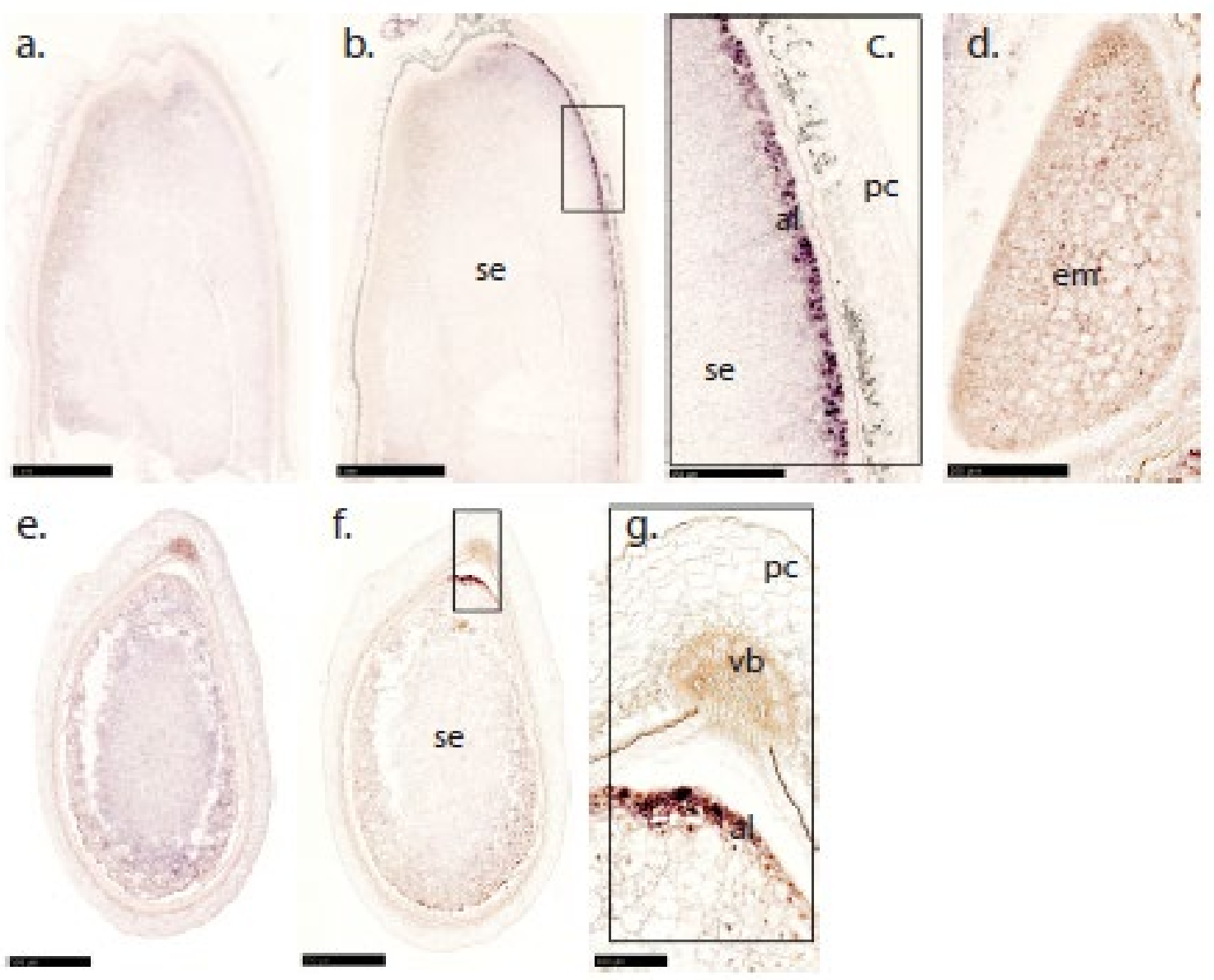

Fig. 4 (a-g). In situ hybridisation of OsIAA29 transcripts in immature rice grains. Hybridisation was done using longitudinal (a-d) and transverse sections (e-g) of immature rice grains at 7 DAP. Images show the absence of in situ hybridisation signal from the sense (a and e) probes and its presence from the anti-sense ( $b-d$ and $f-g$ ) probes. Image $c$ and $g$ are magnified images of marked areas from $b$ and $f$, respectively. Image $\mathrm{d}$ is derived from the same grain as $\mathrm{b}$ and shows the absence of hybridisation signal in the embryo. Sense probes (a and e) were used as negative control in the experimentation. Note the strong hybridisation signal in the dorsal aleurone (b-c and $\mathrm{f}-\mathrm{g}$ ). The dorsal side in longitudinal grain sections can be determined by the position of the major vascular bundles running in the pericarp along the dorsal side or by the position of the embryo located always on the ventral side. In case of transverse sections, the dorsal side is determined by the position of the major vascular bundles. Scale bars: (a and b) $1000 \mu \mathrm{m}$; (c) $250 \mu \mathrm{m}$; (d) $100 \mu \mathrm{m}$; (e and f) $500 \mu \mathrm{m}$; (g) $100 \mu \mathrm{m}$. Abbreviations used for annotation: $\mathrm{al}=$ aleurone; $\mathrm{em}=$ embryo; $\mathrm{pc}=$ pericarp; $\mathrm{se}=$ starchy endosperm; $\mathrm{vb}=$ vascular bundle. 


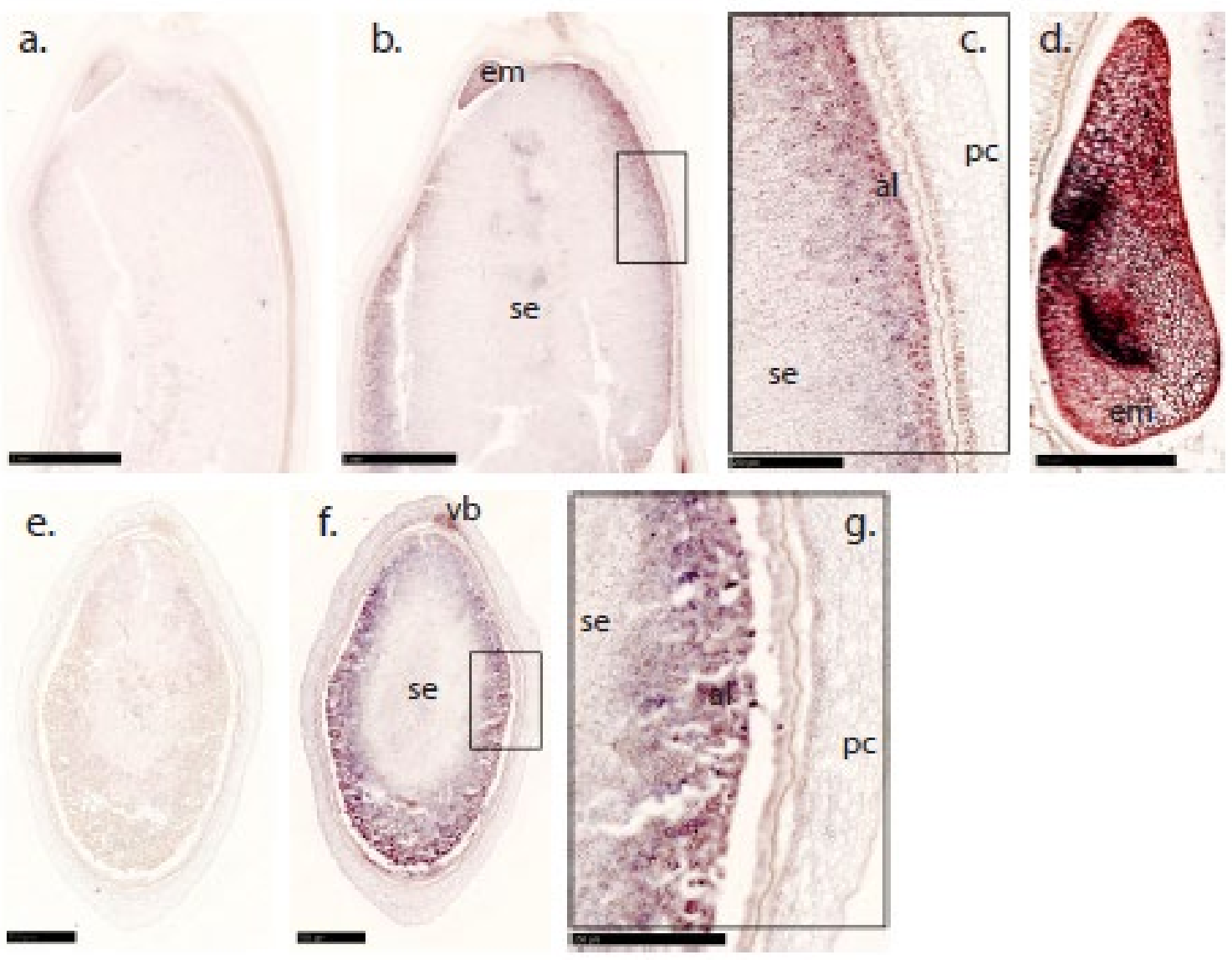

Fig. 5 (a-g). In situ hybridisation of $O_{S} Y U C 12$ transcripts in immature rice grains. Hybridisation was done using longitudinal (a-d) and transverse sections (e-g) of immature rice grains at 7 DAP. Images show the absence of in situ hybridisation signal from the sense (a and e) probes and its presence from the anti-sense ( $b-d$ and $f-g$ ) probes. Image $c$ and $g$ are magnified images of marked areas from $b$ and $f$, respectively. Image $d$ is derived from the same grain as $b$ and shows the presence of hybridisation signal in the embryo. Sense probes (a and e) were used as negative control in the experimentation. Note the strong hybridisation signal in the aleurone, sub-aleurone and embryo (b-d and $\mathrm{f}-\mathrm{g}$ ). The dorsal side in longitudinal grain sections can be determined by the position of the major vascular bundles running in the pericarp along the dorsal side or by the position of the embryo located always on the ventral side. In case of transverse sections, the dorsal side is determined by the position of the major vascular bundles. Scale bars: (a and b) $1000 \mu \mathrm{m}$; (c and d) $250 \mu \mathrm{m}$; (e and f) $500 \mu \mathrm{m}$; (g) 250 $\mu \mathrm{m}$. Abbreviations used for annotation: al = aleurone; em $=$ embryo; $\mathrm{pc}=$ pericarp; $\mathrm{se}=$ starchy endosperm; $\mathrm{vb}=$ vascular bundle. 


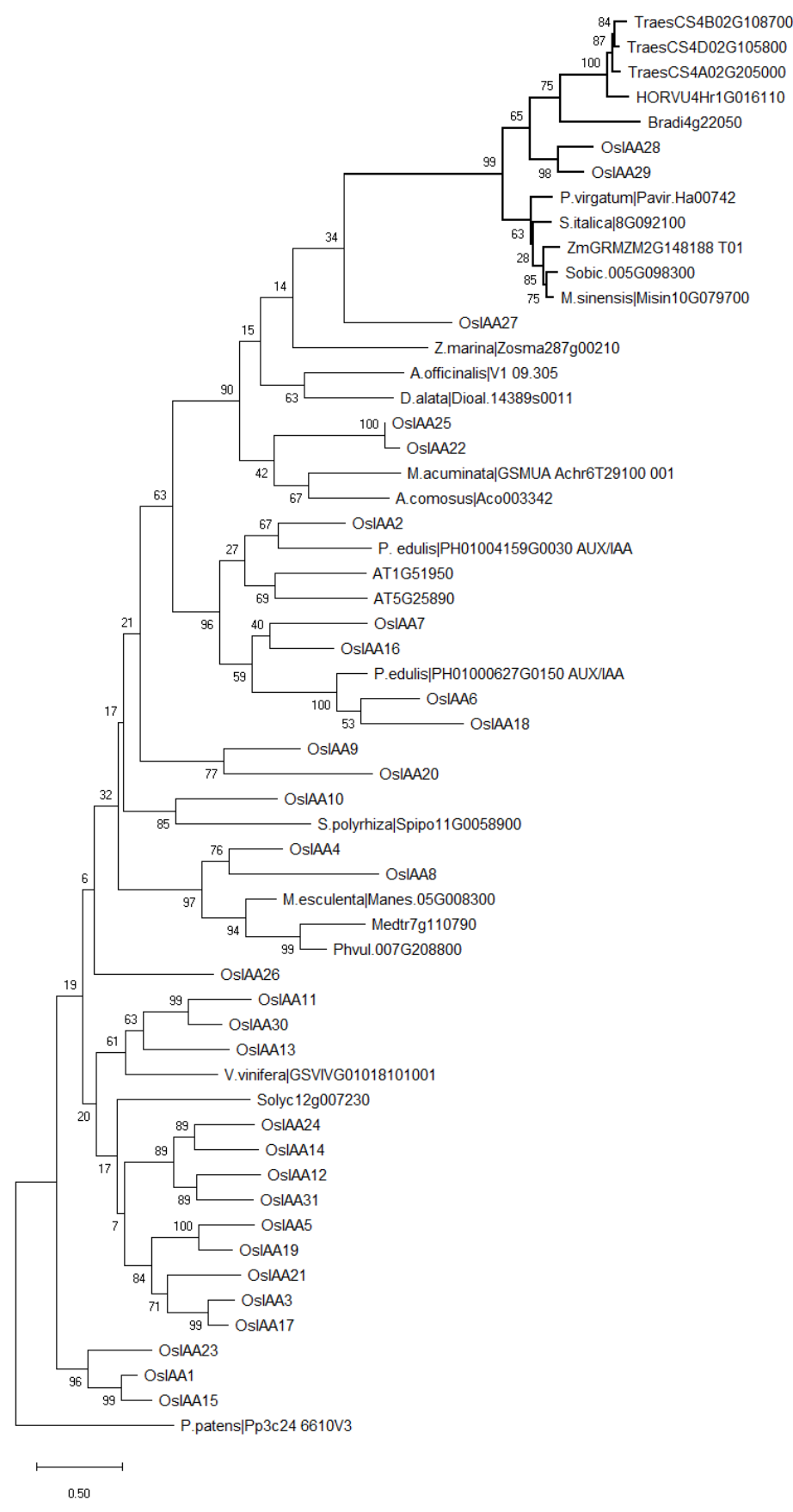

Fig. 6. Phylogenetic tree showing relationship of rice AUX/IAA proteins including OsIAA29 with closest homologous peptides from other grasses, non-grass monocots and eudicots. The phylogram was generated by MEGA 10.0 (Kumar et al. 2018), using the Maximum Likelihood method (Jones et al. 1992). Multiple sequence alignments were done using MUSCLE (Edgar 2004). Bootstrap confidence levels were obtained from 500 replicates (Felsenstein, 1985). Monocots included were: Oryza sativa (rice), Phyllostachys edulis (moso bamboo), Brachypodium distachyon, Hordeum vulgare (barley), Triticum aestivum (wheat), Zea mays (maize), Sorghum bicolor (sorghum), Setaria italica (millet), Panicum virgatum (switchgrass), Miscanthus sinensis, Musa acuminata (banana), Ananas comosus (pineapple), Dioscorea alata (purple yam), Zostera marina (a sea grass), Spirodela polyrhiza (a duckweed) and Asparagus officinalis (asparagus). Eudicots included were: Arabidopsis thaliana, Medicago truncatula, Phaseolus vulgaris (common bean), Solanum lycopersicum (tomato), Vitis vinifera (grape) and Manihot esculenta (cassava). The tree is rooted with a sequence from the moss Physcomitrella patens. Putative orthologues of OsIAA29 are shown in bold. Scale bar $=0.50$ denotes amino acid substitutions per site. 


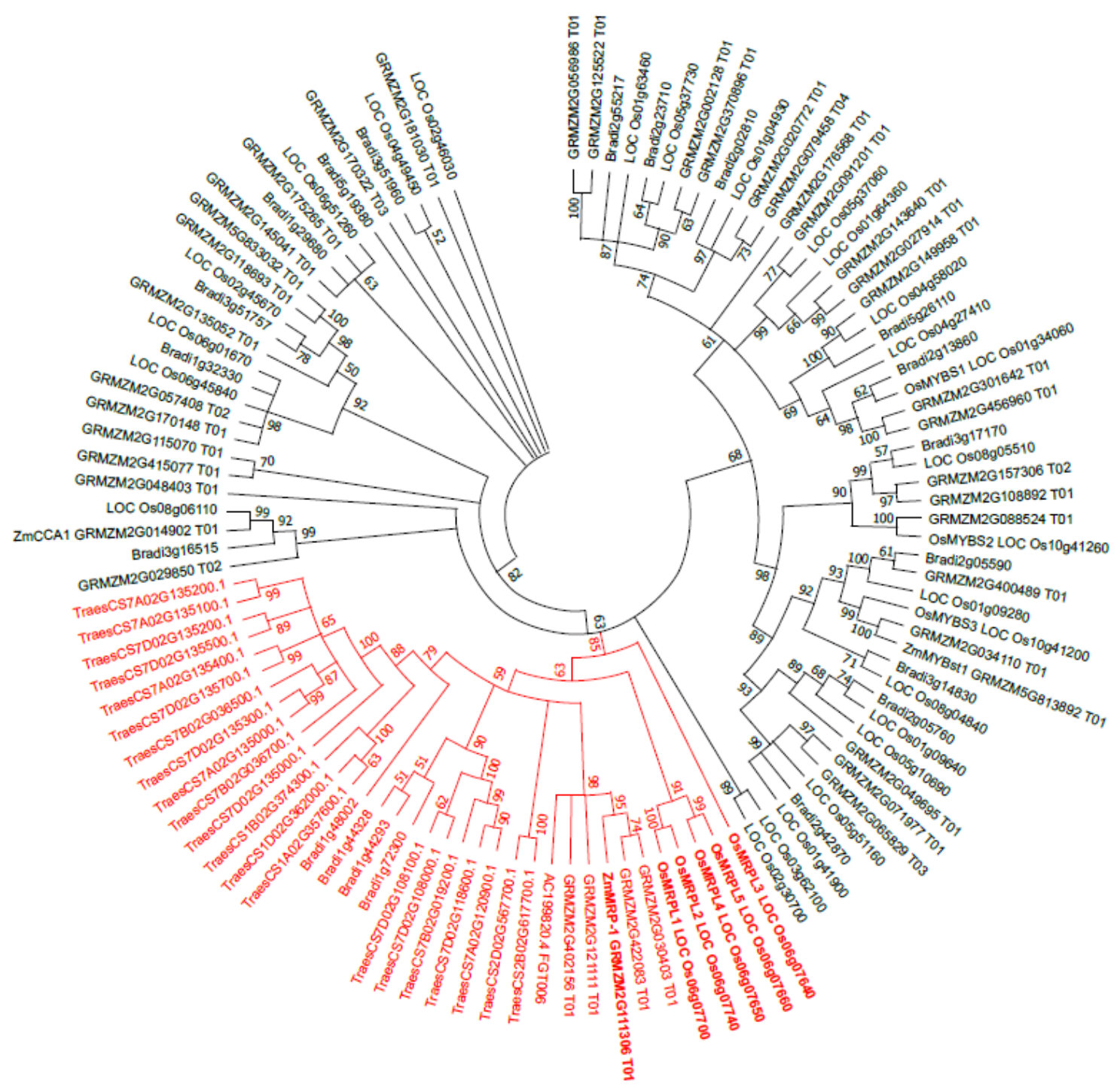

Fig. 7. Phylogenetic tree showing the relationships between CCA1-like MYB-related proteins from maize, rice, Brachypodium distachyon. and wheat. Due to the large number of wheat proteins, only those in the ZmMRP-1 clade are shown. The consensus phylogram was generated by MEGA 10.0 (Kumar et al. 2018), using the Maximum Likelihood method (Jones et al. 1992). Multiple sequence alignments were done using MUSCLE (Edgar 2004). Bootstrap confidence levels were obtained from 500 replicates (Felsenstein, 1985). The cut-off value for the consensus tree was set at 50 . The clade containing ZmMRP-1 is shown in red. 


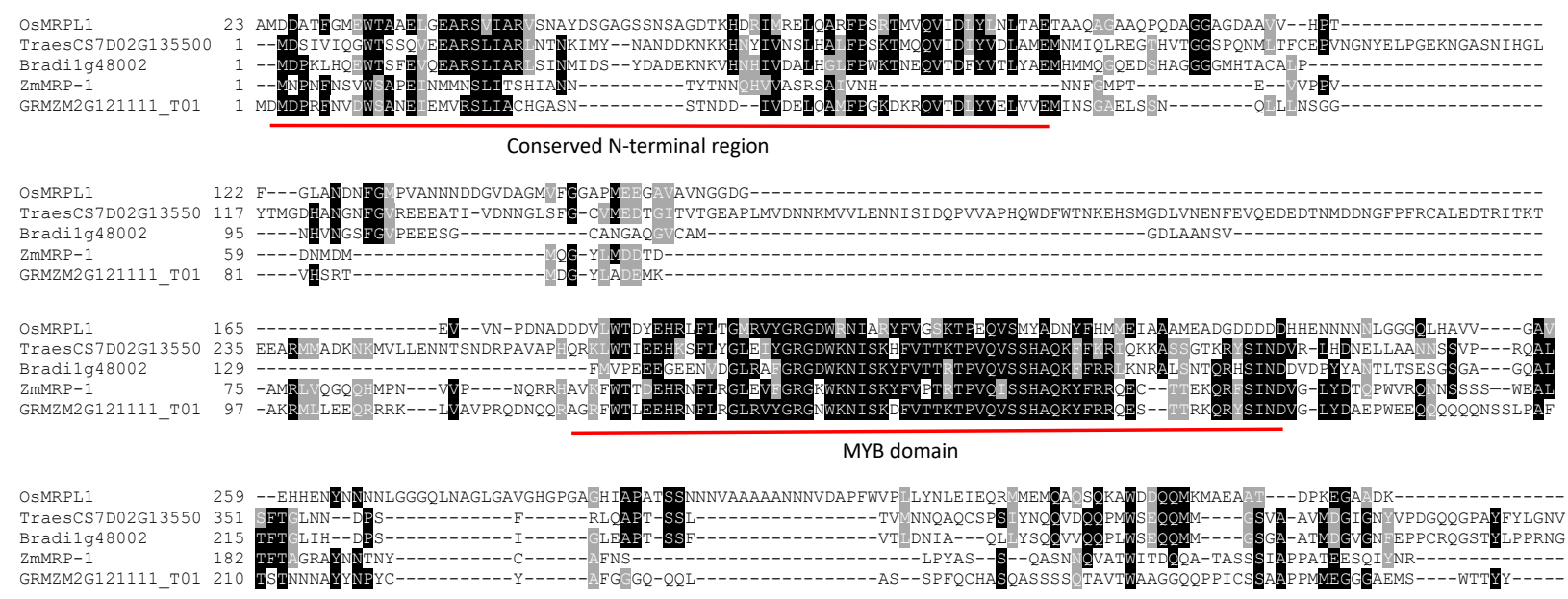

Fig. 8 Multiple sequence alignment comparing ZmMRP-1 and one other MRP-1 like protein from maize encoded by GRMZM2G121111_T01 with putative orthologues from rice (OsMRPL1), wheat (TraesCS7D02G135500.1), B. distachyon (Bradi1g48002). The alignment was carried out in CLUSTAL Omega accessed via https://www.ebi.ac.uk and displayed using Boxshade (https://embnet.vital-it.ch/software/BOX_form.html). Shaded symbols indicate amino acids conserved in at least $50 \%$ of sequences. 


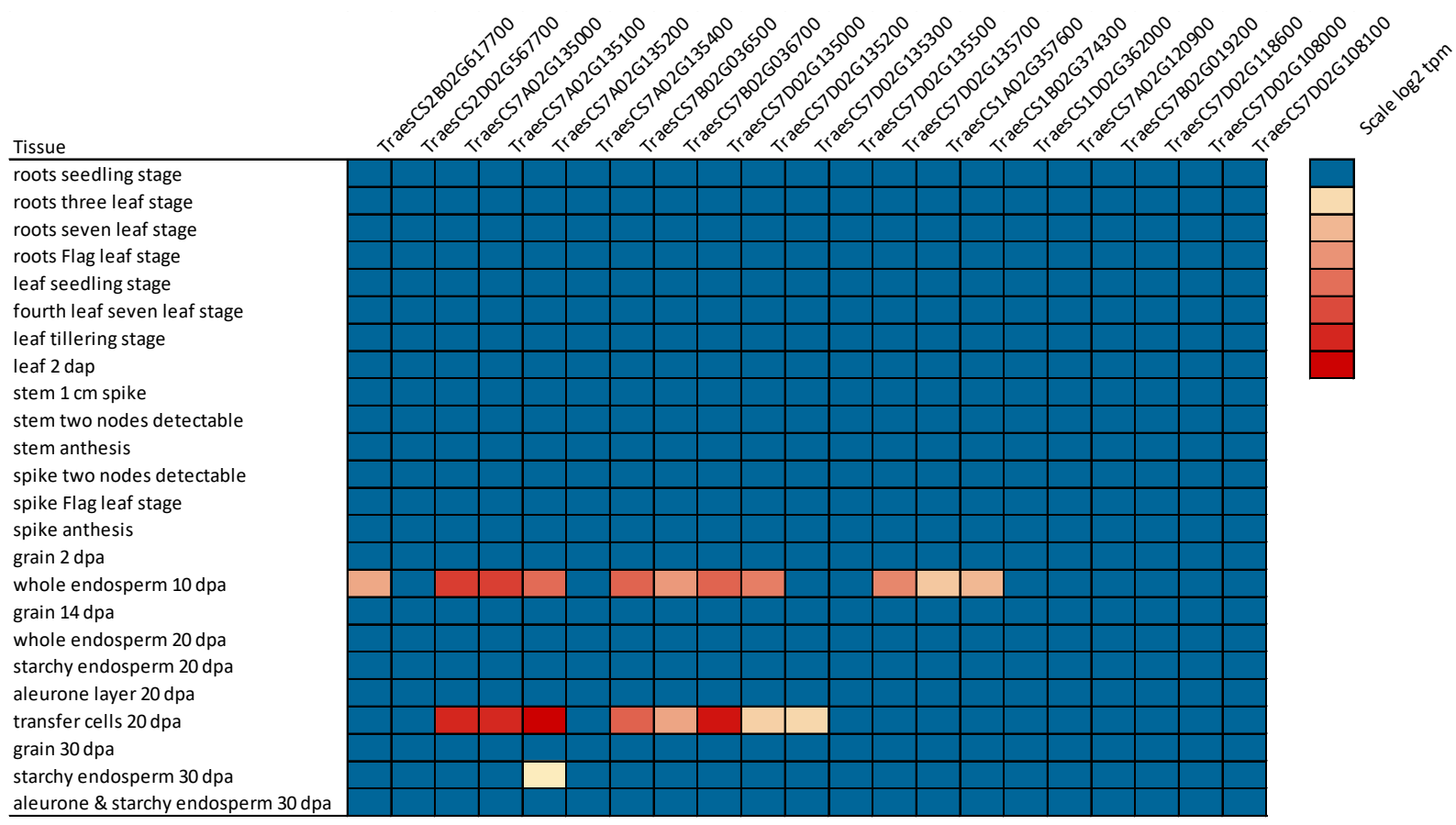

Fig. 9. Heat map showing expression of putative wheat orthologues of ZmMRP-1 in different vegetative and reproductive tissues. RNA-seq data is derived from two studies, Wheat development time course (Choulet et al. 2014) and Grain development time course (Pfeifer et al. 2014), both studies using the Chinese Spring variety and accessed via the Wheat Expression Browser (Borrill et al. 2016; Ramírez-González et al. 2018). Gene expression is expressed as log2 TPM (Transcripts Per Million). 


\section{Supplementary material}

Suppl. Table S1: Primer pairs used for qRT-PCR. F=forward primers; R=reverse primers.

\begin{tabular}{|c|c|c|c|}
\hline MSU7 Locus ID & Gene name & Primer sequence $\left(5^{\prime}-3^{\prime}\right)$ & $\begin{array}{l}\text { Product } \\
\text { size (bp) }\end{array}$ \\
\hline \multirow[t]{2}{*}{ LOC_Os02g17230 } & OsYUC12 & F: GAGTTATCGACGTGCTCGAA & 147 \\
\hline & & R: TGCTTTCACCATTCTTTAGCC & \\
\hline \multirow[t]{2}{*}{ LOC_Os11g11430 } & OsIAA29 & F: CAACTATTCCATGTCACCATTTG & 131 \\
\hline & & R: ATGGGACATCACCAAGGAAG & \\
\hline \multirow[t]{2}{*}{ LOC_Os01g28474 } & $A L 1$ & F: GCATGCCAAGCCTCATAAGT & 100 \\
\hline & & R: GCTGAGGTGAGTTACTTGTACG & \\
\hline \multirow[t]{2}{*}{ LOC_OsO6g07740 } & OsMRPL1 & F: CGATGTGCTTTGGACCGATT & 137 \\
\hline & & R: TATCGGCGTACATGGAGACC & \\
\hline \multirow[t]{2}{*}{ LOC_Os06g07640 } & OsMRPL3 & F: ACAGAGGAAGAACACAGGCT & 131 \\
\hline & & R: AAGAACTTCTGCGCGTGG & \\
\hline \multirow[t]{2}{*}{ LOC_Os06g07650 } & OsMRPL4 & F: GTGGTGCCGGTGGTGATG & 117 \\
\hline & & R: CCTGTGCTCTTCAGTGGTC & \\
\hline \multirow[t]{2}{*}{ LOC_Os01g58660 } & OsPR602 & F: TGCATGGAGAAGGTCGTGTA & 130 \\
\hline & & R: AGCCATCATCTTCATGCTTGA & \\
\hline \multirow[t]{2}{*}{ LOC_Os02g39625 } & OsPR9a & F: TATGCAGCCGATGAAGGGA & 100 \\
\hline & & R: ACGGAGCATTGTCAGGGTAA & \\
\hline \multirow[t]{2}{*}{ LOC_Os02g42314 } & OsUBC & F: CTGCGAGCTGAAACACTTTG & 105 \\
\hline & & R: TTCTCGCTGCACCTCCTTAT & \\
\hline
\end{tabular}


bioRxiv preprint doi: https://doi.org/10.1101/2021.03.04.434009; this version posted March 5, 2021. The copyright holder for this preprint (which was not certified by peer review) is the author/funder. All rights reserved. No reuse allowed without permission.

Suppl. Table S2: Primer pairs used for probe preparation. $\mathrm{F}=$ forward primers; $\mathrm{R}=$ reverse primers.

\begin{tabular}{|c|c|c|c|}
\hline MSU7 Locus ID & Gene name & Primer sequence $\left(5^{\prime}-3^{\prime}\right)$ & $\begin{array}{l}\text { Product } \\
\text { size (bp) }\end{array}$ \\
\hline \multirow[t]{2}{*}{ LOC_Os02g17230 } & OsYUC12 & F: TGGAGGAGGTGGTTGTTCTC & 988 \\
\hline & & R: ATGCTTTCACCATTCTTTAGCC & \\
\hline \multirow[t]{2}{*}{ LOC_Os11g11430 } & OsIAA29 & F: GCCTATTGTGGTCAGCTTTCA & 817 \\
\hline & & R: CCCCTGGTCAGGAGTAACAA & \\
\hline \multirow[t]{2}{*}{ LOC_Os01g58660 } & OsPR602 & F: CACCACAACAATATAGCCACTGT & 527 \\
\hline & & R: GGCCACAATTCCCCACAAAT & \\
\hline
\end{tabular}



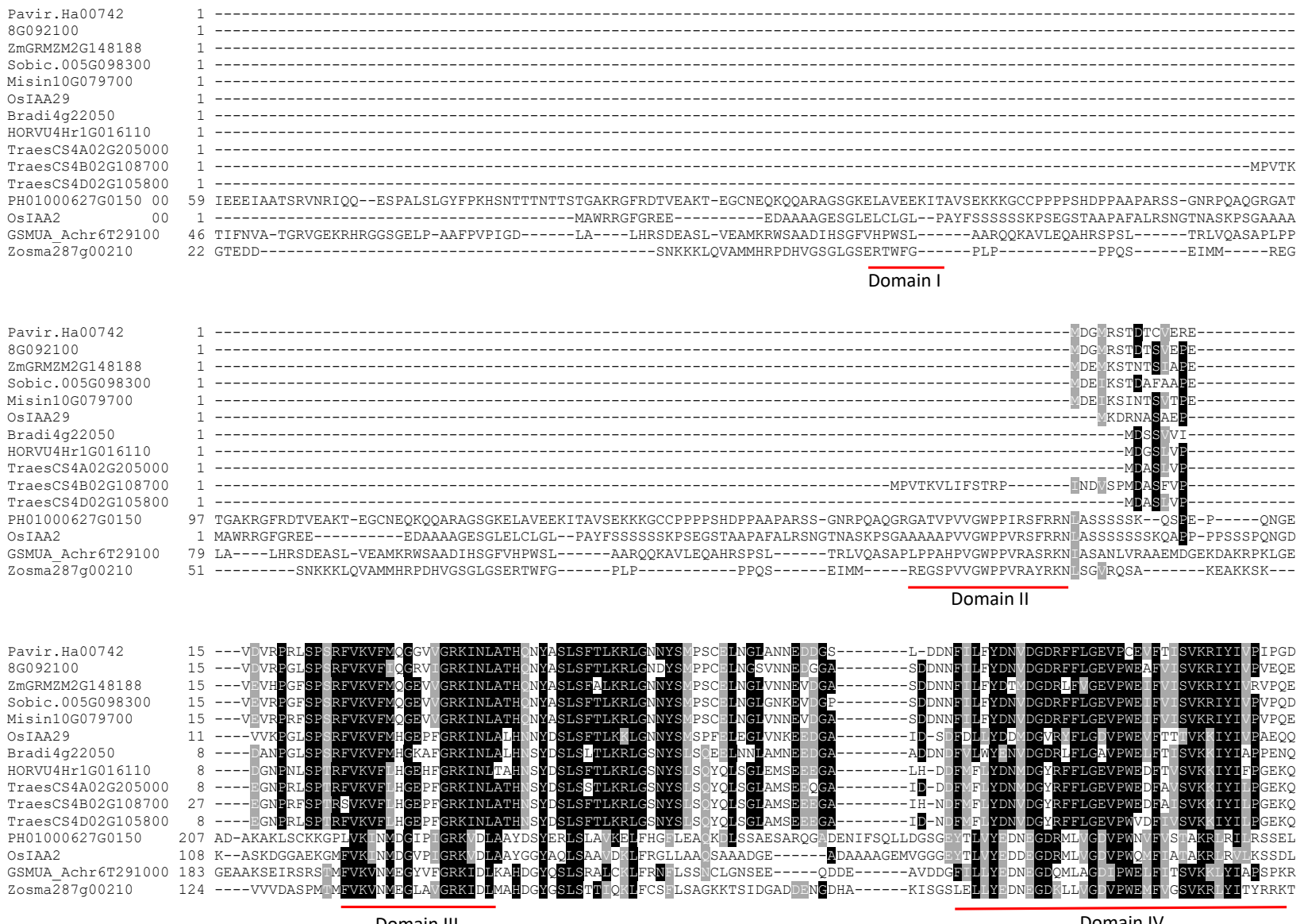

Domain III
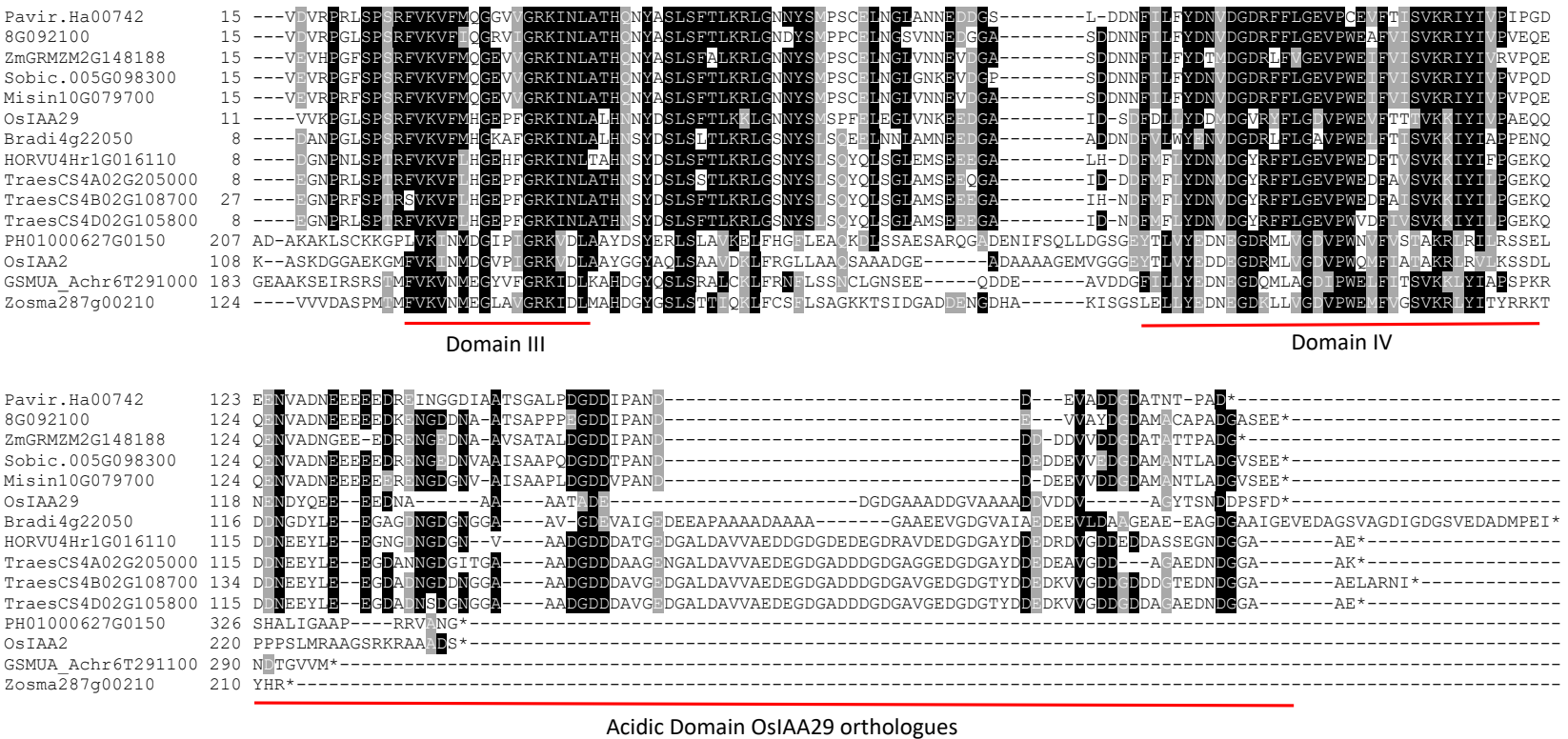

Suppl. Fig. S1. Multiple sequence alignment of OsIAA29, with putative orthologues from other cereals (Pavir.Ha00742 Panicum virgatum, 8G092100 Setaria italica, ZmGRMZM2G148188_T01 maize, Sobic.005G098300 sorghum, Misin10G079700 Miscanthus sinensis, Bradi4g22050 Brachypodium distachyon, HORVU4Hr1G016110 barley, TraesCS4B02G108700 etc wheat) and the closest homologous proteins from moso bamboo (PH01000627G0150), banana (GSMUA_Achr6T29100_001) and Zostera marina Zosma287g00210.1, as well as canonical AUX/IAA protein OsIAA2. The alignment was carried out in CLUSTAL Omega accessed via https://www.ebi.ac.uk and displayed using Boxshade (https://embnet.vitalit.ch/software/BOX_form.html). Shaded symbols indicate amino acids conserved in at least $50 \%$ of sequences. 
A.

\section{HORVU4Hr1G016110}

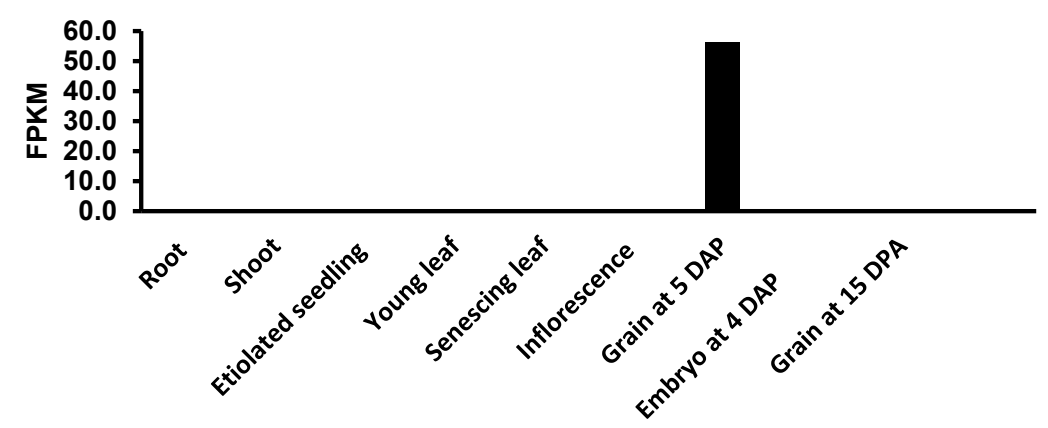

B.

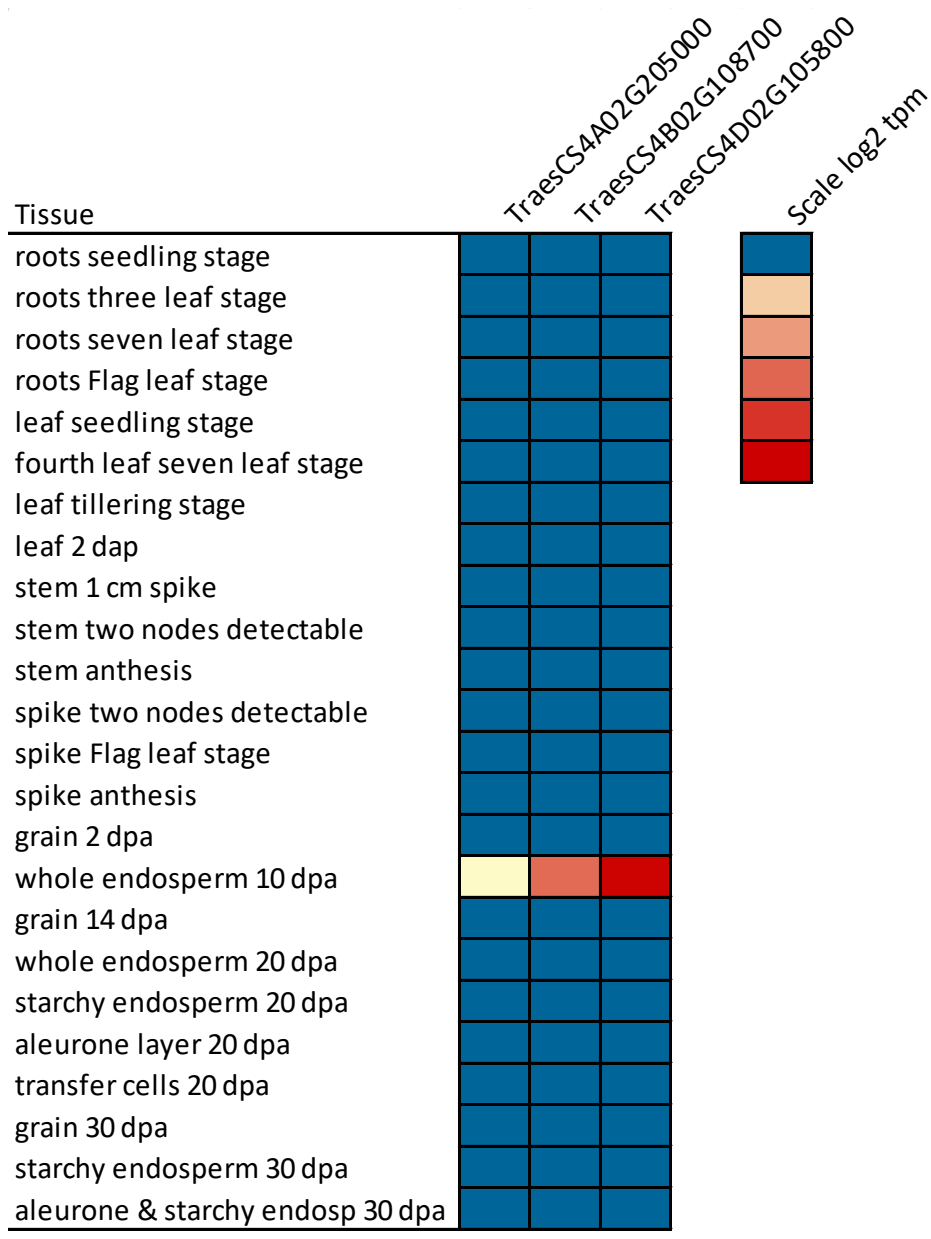

Suppl. Fig. S2. Expression of barley and wheat orthologues of OsIAA29. (A) RNA-seq data for expression of the barley orthologue (HORVU4Hr1G016110) retrieved from BaRTv1.0 (RapazoteFlores et al. 2019). FPKM = Fragments Per Kilobase of transcript per Million mapped reads. (B) Heat map showing expression of the three wheat orthologues of OsIAA29 in different vegetative and reproductive tissues. RNA-seq data is derived from two studies, Wheat development time course (Choulet et al. 2014) and Grain development time course (Pfeifer et al. 2014), both studies using the Chinese Spring variety and accessed via the Wheat Expression Browser (Borrill et al. 2016; RamírezGonzález et al. 2018). Gene expression is expressed as $\log 2$ TPM (Transcripts Per Million). 
bioRxiv preprint doi: https://doi.org/10.1101/2021.03.04.434009; this version posted March 5, 2021. The copyright holder for this preprint (which was not certified by peer review) is the author/funder. All rights reserved. No reuse allowed without permission.

CLUSTAL O(1.2.4) multiple sequence alignment

LOC_Os0 $6 g 07640$
LOC_Os0 6907650
LOC_Os0 6907660
LOC_Os0 6907700
LOC_Os0 6907740

LOC_Os06g07640 LOC-Os06g07650 LOC_Os06g07660 LOC_Os06g07700 LOC_Os06g07740

LOC Os06g07640 LOC_Os06g07650 LOC Os06g07660 LOC_Os06g07700 LOC_Os06g07740

LOC Os06g07640 LOC Os06g07650 LOC_Os06g07660 LOC-Os06g07700 LOC_Os06g07740

LOC_Os06g07640 LOC_Os06g07650 LOC Os06g07660 LOC_Os06g07700 LOC-Os06g07740

LOC Os06g07640 LOC_Os06g07650 LOC Os06g07660 LOC Os06g07700 LOC_Os06g07740

LOC Os06g07640 LOC Os06g07650 LOC_Os06g07660 LOC ${ }^{-}$Os0 0607700 LOC_Os06g07740

LOC_Os06g07640 LOC Os06g07650 LOC Os06g07660 LOC_Os06g07700 LOC_Os06g07740

LOC Os06g07640 LOC_Os06g07650 LOC Os06907660 LOC Os06g07700 LOC_Os06g07740

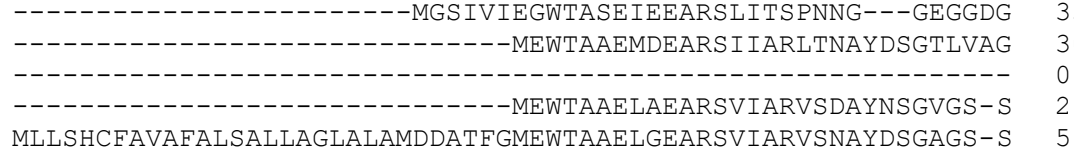

EGNKQKHCGH IVMELHEWFPWKTIGQVIGLYMKLNAGKPMVMHS LNKS DANNS I---GEV AGNGDTRHDRIVRELQAWLPWRTMDQLIGLYIELMAEEPAAA---QPQY----FDAGAVV

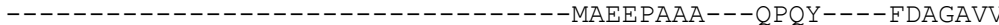
SSACDTKHDRIMRELQARFPSRTMVQVIDLYVNLTVETAA-----QPQDAGSAGDAAAVV NSAGDTKHDRIMRELQARFPSRTMVQVIDLYLNLTAETAAQAGAAQPQDAGGAGDA-AVV

$$
:
$$

DHVSALANGN------PVRL---EEHR---PMLNNVGLVFDYPL---------EEMEMEN DPTFDFFNDHNNFLGMP P P PVQQADDHNMNNVVADAGMNYYYGGGGAGGAMVFGGAPMGE DPTFDFFIDHNNFLGMP P PPVQQADDHAMNNVVADASMNYFYGGGA---MVFGGGAPMGE HPTFA--------GGMPVVN---NN--------------------D-GMVHGGAAMEV HPTFGLANDN---FGMPVAN---NND---------------DGVDA-GMVFGGAPMEE $\cdot$

QTDQ-EPKMVVEEEVQPKEGLVIKEKEAGVSKIHTNSQHVTPS IKRRVIWTEEEHR---TVEQAAPPVP----V---VPVVMNRDDDEVNNQGGGRHRAAPTNTTRRFWTTEEHR---TVEQAAPPVPAPVAV---APVVMNRDDDEVNNQGGRRHRAAPTNTTRRFWTTEEHRATLI

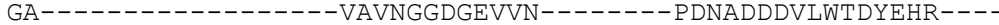
GA----------------VAVNGGDGEVVN--------PDNADDDVLWTDYEHR---$\therefore:: \quad * \quad: \quad: * \star \quad \star \star \star$

QFLRGLRVYGRGEWKS ISMNFVRSKTPV YSSYDFDPPITNLIITMTRMKKNMYFLCETMQFLRGLRVYGRGEWKS ISMNFVRSKTPV (1)

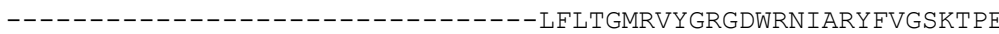
${ }^{\star}:{ }^{\star}:{ }^{*}:{ }^{* *}:{ }^{\star}:{ }^{\star}: \quad:{ }^{*}::^{*}$

OVSSHAQKFFLKMEARGEAVPPPAKR------RRRRITGDQQAA---AAEHAAA------ 257 QVSSHAQKYFRRVESAAADKQRYSINDVGL--------NDDTAAMDGTNSYSNNNF 268 QVSSHAQKYFRRVESAAADKQRYSINDVGL-----------NDDTAAMDGTNSYSNNNF 241 QISMYADNYFHMMEIAAAMEADGGDDDDGHHEI-----------------NNNNNNL 208 QVSMYADNYFHMMEIAAAMEADGDDDDDHHENNNNNLGGGQLHAVVGAVEHHENYNNNNL 272 ${ }^{\star}:{ }^{\star}:{ }^{\star}:::^{\star} \quad:{ }^{\star}$.

-------------------LRRRMPV----------PPPPFNPFLLPSLVAPVMH GGWQSLAFAGGHLEPVSGGGAARQVIAPASSS-------AAAMNSAAQFWAPMLFNPQIQ GGWQSLAFAGGHLEPVSGGGAARQVIAPASSS------AAAMNSAAQFWAPMLFNPQIQ GGGQLHAVVGA-----VGHGPGAGHIAPATPSNNNTAAAAVNNNVDTPFWVPLLYNPEIE GGGQLNAGLGA-----VGHGPGAGHIAPATS SNNNVAAAAANNNVDAPFWVPLLYNLEIE $\star$. $* * * \quad:$

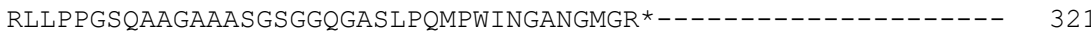
QQFMQM-----------QAQTQQAWNDQHMMMAAAP---MEGATDTNFEPAGAVNY 363 QQFMQM-------------QAQTQQAWNDQHMMMAAAP---MEGATDTNFEPAGAVNY 336 QRMMEM--------------QAQSQKAWDDQQMKMAEAATPKEEGAADK*-------- 298

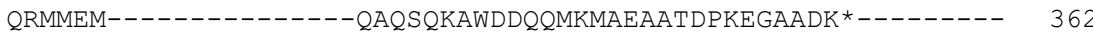

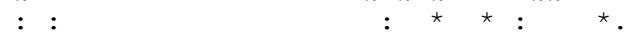

$-----321$

YYYQQQQEEEEGGAYGVPADQWMMNQNNNMC* 394 YYYQQQQEEEEGGAYGVPADQWMMNQNNNMF* 367

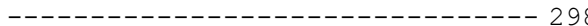

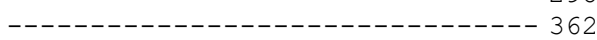

Suppl. Fig. S3 Multiple sequence alignment comparing OsMRPL proteins The alignment was carried out in CLUSTAL Omega accessed via https://www.ebi.ac.uk. 


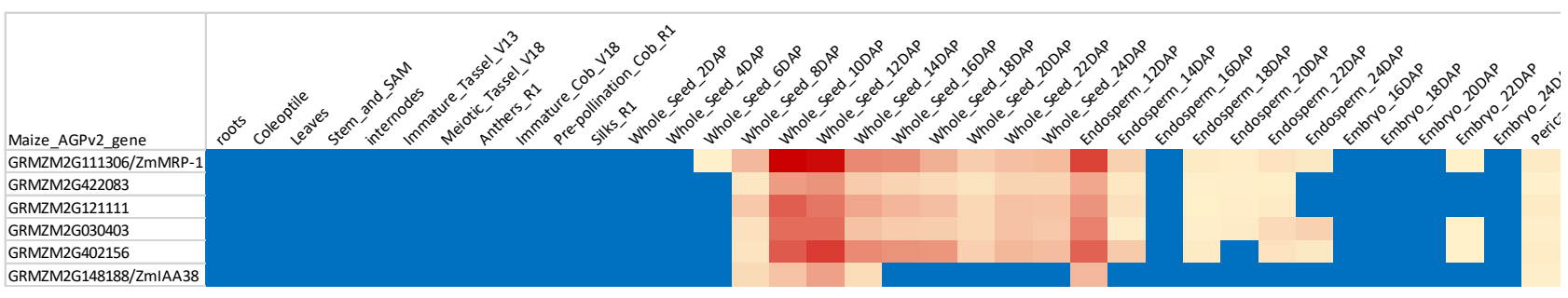

Suppl. Fig. S4. Comparing expression of ZmMRP-1 and related genes with that of ZmIAA38, the maize orthologue of OsIAA29 using RNA-seq data from Stelpflug, et al. (2016). As no expression of any genes was seen in any of the root, leaf, stem or internode samples, a single sample of each only was included in this figure. 